\title{
Composition of the Rex Chert and Associated Rocks of the Permian Phosphoria Formation: Soda Springs Area, SE Idaho
}

B $y$

James R. Hein ${ }^{1}$, Brandie McIntyre ${ }^{1}$, Robert B. Perkins ${ }^{1}$, David Z. Piper $^{1}$, James Evans ${ }^{2}$

\section{Open-File Report 02-345}

2002

Prepared in Collaboration With

U.S. Bureau of Land Management

U.S. Forest Service

Agrium U.S. Inc.

Astaris LLC

J.R. Simplot Company

Rhodia Inc.

Monsanto Co.

This report is preliminary and has not been reviewed for conformity with U.S. Geological Survey editorial standards or with the North American Stratigraphic Code. Any use of trade, firm, or product names is for descriptive purposes only and does not imply endorsement by the U.S. Government.

\section{U. S. DEPARTMENT OF THE INTERIOR}

U. S. GEOLOGICAL SURVEY

${ }^{1}$ U.S. Geological Survey, 345 Middlefield Rd., Menlo Park, CA, 94025

${ }^{2}$ U.S. Geological Survey, 904 W. Riverside Ave., Spokane, WA, 99201 


\section{CONTENTS}

ABSTRACT

$\begin{array}{ll}\text { INTRODUCTION } & 5\end{array}$

Background 5

Location and General Geology 5

$\begin{array}{ll}\text { Lithostratigraphy } & 7\end{array}$

METHODS 9

$\begin{array}{lr}\text { Field Sampling } & 9\end{array}$

$\begin{array}{ll}\text { Rock-Sample Preparation } & 10\end{array}$

$\begin{array}{ll}\text { Analyses } & 10\end{array}$

$\begin{array}{lr}\text { RESULTS } & 16\end{array}$

$\begin{array}{ll}\text { Petrography } & 16\end{array}$

$\begin{array}{ll}\text { Mineralogy } & 18\end{array}$

$\begin{array}{lr}\text { Chemical Composition } & 18\end{array}$

Stratigraphic Changes in Chemical Composition 23

$\begin{array}{ll}\text { Phase Associations of Elements } & 23\end{array}$

$\begin{array}{ll}\text { DISCUSSION AND CONCLUSIONS } & 28\end{array}$

ACKNOWLEDGMENTS

REFERENCES CITED 


\section{FIGURES}

Figure 1. Index map of SE Idaho and locations of sections of Rex Chert.

Figure 2. Three measured stratigraphic sections of the Rex Chert Member of the Phosphoria Formation.

Figure 3. Q-mode rotated factor scores and phase association of elements for chemical data from sections 1,5 , and 7 .

Figure 4. Q-mode rotated factor scores and phase association of elements for chemical data from section 1 .

Figure 5. Q-mode rotated factor scores and phase association of elements for chemical data from section 7 .

\section{TABLES}

Table 1. GPS locations of Rex Chert sections in the vicinity of Dry Valley, SE Idaho

Table 2. X-ray diffraction mineralogy of samples of the Rex Chert, cherty shale, and Meade Peak Members of the Phosphoria Formation

Table 3. Chemical Composition of samples from the Rex Chert, cherty shale, and Meade Peak Members of the Phosphoria Formation

Table 4. Statistics of chemical data for 39 Rex Chert and cherty shale samples from sections 1,5 , and 7

Table 5. Statistics of chemical data for 14 Rex Chert and cherty shale samples, section 1

Table 6. Statistics of chemical data for 4 Rex Chert samples, section 5

Table 7. Statistics of chemical data for 21 Rex Chert samples, section 7

Table 8. Correlation coefficient matrix of Rex Chert and cherty shale member chemical data combined from measured sections 1,5 , and 7

Table 9. Correlation coefficient matrix of Rex Chert and cherty shale members chemical data from measured section 1

Table 10. Correlation coefficient matrix of Rex Chert Member chemical data from measured section 7 


\section{ABSTRACT}

This study, one in a series, reports bulk chemical and mineralogical compositions, as well as petrographic and outcrop descriptions of rocks collected from three measured outcrop sections of the Rex Chert member of the Phosphoria Formation in SE Idaho. The three measured sections were chosen from ten outcrops of Rex Chert that were described in the field. The Rex Chert overlies the Meade Peak Phosphatic Shale Member of the Phosphoria Formation, the source of phosphate ore in the region. Rex Chert removed as overburden comprises part of the material disposed in waste-rock piles during phosphate mining. It has been proposed that the chert be used to cap and isolate waste piles, thereby inhibiting the leaching of potentially toxic elements into the environment. It is also used to surface roads in the mining district. The rock samples studied here constitute a set of individual chert beds that are representative of each stratigraphic section sampled. The informally named cherty shale member that overlies the Rex Chert in measured section 1 was also described and sampled. The upper Meade Peak and the transition zone to the Rex Chert were described and sampled in section 7 . The cherts are predominantly spicularite composed of granular and mosaic quartz, and sponge spicules, with various but minor amounts of other fossils and detrital grains. The cherty shale member and transition rocks between the Meade Peak and Rex Chert are siliceous siltstones and argillaceous cherts with ghosts of sponge spicules and somewhat more detrital grains than the chert. The overwhelmingly dominant mineral is quartz, although carbonate beds are rare in each section and are composed predominantly of calcite and dolomite in addition to quartz. Feldspar, mica, clay minerals, calcite, dolomite, and carbonate fluorapatite are minor to trace minerals in the chert.

The mean concentrations of oxides and elements in the Rex Chert and the cherty shale member are dominated by $\mathrm{SiO}_{2}$, which averages $94.6 \%$. Organic-carbon contents are generally very low in the chert, but are up to $1.8 \mathrm{wt}$. \% in cherty shale member samples and up to $3.3 \%$ in samples from the transition between the Meade Peak and Rex Chert. Likewise, phosphate $\left(\mathrm{P}_{2} \mathrm{O}_{5}\right)$ is generally low in the chert, but can be up to $3.1 \%$ in individual beds. Selenium concentrations in Rex Chert and cherty shale member samples vary from $<0.2$ to $138 \mathrm{ppm}$, with a mean concentration of $7.0 \mathrm{ppm}$. This mean Se content is heavily dependent on two values of 101 and $138 \mathrm{ppm}$ for rocks from the transition zone between the Meade Peak and Rex Chert. Without those two samples, the mean Se concentration would be $<1.0 \mathrm{ppm}$. Other elements of environmental interest, As, $\mathrm{Cr}, \mathrm{V}$, $\mathrm{Zn}, \mathrm{Hg}$, and $\mathrm{Cd}$, are generally near or below their content in average continental shale. Stratigraphic changes (equivalent to temporal changes in the depositional basin) in chemical composition of rocks are notable either as uniform changes through the sections or as distinct differences in the mean composition of rocks that compose the upper and lower halves of the sections.

Q-mode factors are interpreted to represent the following rock and mineral components: chert-silica component consisting of $\mathrm{Si}( \pm \mathrm{Ba})$; phosphorite-phosphate component composed of P, Ca, As, Y, V, Cr, Sr, and La ( \pm Fe, Zn, Cu, Ni, Li, Se, Nd, Hg); shale component composed of $\mathrm{Al}, \mathrm{Na}, \mathrm{Zr}, \mathrm{K}, \mathrm{Ba}, \mathrm{Li}$, and organic $\mathrm{C}( \pm \mathrm{Ti}, \mathrm{Mg}, \mathrm{Se}, \mathrm{Ni}, \mathrm{Fe}, \mathrm{Sr}$, $\mathrm{V}, \mathrm{Mn}, \mathrm{Zn}$ ); carbonate component (dolomite, calcite, silicified carbonates) composed of carbonate $\mathrm{C}, \mathrm{Mg}, \mathrm{Ca}$, and $\mathrm{Si}( \pm \mathrm{Mn})$; tentatively organic matter-hosted elements (and/or sulfide-sulfate phases) composed of $\mathrm{Cu}$ ( \pm organic $\mathrm{C}, \mathrm{Zn}, \mathrm{Mn} \mathrm{Si}, \mathrm{Ni}, \mathrm{Hg}$, and $\mathrm{Li}$ ). Selenium shows a dominant association with the shale component, but correlations and Qmode factors also indicate that organic matter (within the shale component) and carbonate fluorapatite may host a portion of the Se. Consideration of larger numbers of factors in Qmode analysis indicates that native Se (a factor containing Se $( \pm \mathrm{Ba})$ ) may also comprise a minor component of the Se compliment. 


\section{INTRODUCTION}

We have initiated a study of the Rex Chert, which conformably overlies the Meade Peak Phosphatic Shale Member of the Permian Phosphoria Formation. It comprises part of the overburden that is removed to reach the phosphate ore at mines in SE Idaho and therefore comprises a part of the waste dumps. In addition, the Rex Chert is used to surface roads in the mining district. It has been proposed that the chert be used to cap and isolate waste dumps to prevent the release of selenium (Se) and other potentially toxic elements to the environment. Previous spot analyses indicated that Se might occur in high concentrations in some chert beds, so we sampled several outcrop sections to determine the composition of the Rex Chert. A parallel report has been produced by Herring et al. (2002) on channel samples through the Rex Chert from the active Rasmussen Ridge and Enoch Valley phosphate mines. Those channel samples comprise a continuous composite record of Rex Chert composition through the entire section. In contrast, our outcrop samples provide the compositions of a series of individual beds through each outcrop section, with intervening beds being unsampled. One outcrop section sampled also includes the overlying, informally named, cherty shale member of the Phosphoria Formation and another section includes the upper part of the Meade Peak Phosphatic Shale Member and the transition zone between the Meade Peak and Rex Chert.

\section{Background}

U.S. Geological Survey (USGS) geologists have studied the Phosphoria Formation in SE Idaho and the Western U.S. Phosphate Field throughout much of the twentieth century. In response to a request by the U.S. Bureau of Land Management (BLM), a new series of resource and geoenvironmental studies was initiated by the USGS in 1998. Present studies involve many scientific disciplines within the USGS and consist of: (1) integrated, multidisciplinary research directed toward resource and reserve estimations of phosphate in selected 7.5-minute quadrangles; (2) element residences, mineralogical and petrochemical characteristics; (3) mobilization and reaction pathways, transport, and disposition of potentially toxic trace elements associated with the occurrence, development, and use of phosphate rock; (4) geophysical signatures; and (5) improving our understanding of the depositional origin and evolution of the deposit.

To carry out these studies, the USGS formed cooperative research relationships with the BLM and the U.S. Forest Service (USFS), which are responsible for land management and resource conservation on public lands, and with five private companies currently leasing or developing phosphate resources in southeastern Idaho. These companies include $\mathrm{Nu}$-West (Agrium U.S. Inc.) (Rasmussen Ridge mine), Astaris Production LLC (Dry Valley mine), Rhodia Inc. (Wooley Valley mine-inactive), J.R. Simplot Co. (Smoky Canyon mine), and P4 Production LLC (Monsanto and Solutia) (Enoch Valley mine). Because raw data acquired during the project will require time to interpret, data are released in open-file reports for prompt availability to other workers. The open-file reports associated with this series of resource and geoenvironmental studies are submitted to each of the Federal and industry collaborators for technical comment; however, the USGS is solely responsible for the data contained in the reports.

\section{Location and General Geology}

Ten sections of Rex Chert located in the vicinity of Dry Valley, northeast of Soda Springs in SE Idaho (Fig. 1; Table 1), were described in the field. Three of those sections $(1,5,7)$ were measured (Figs. 2-4) and sampled in detail for chemical, mineralogical, and petrographic analyses. This region of SE Idaho has supported extensive phosphate mining over the past several decades and currently has four active mines. Service (1966) evaluated 


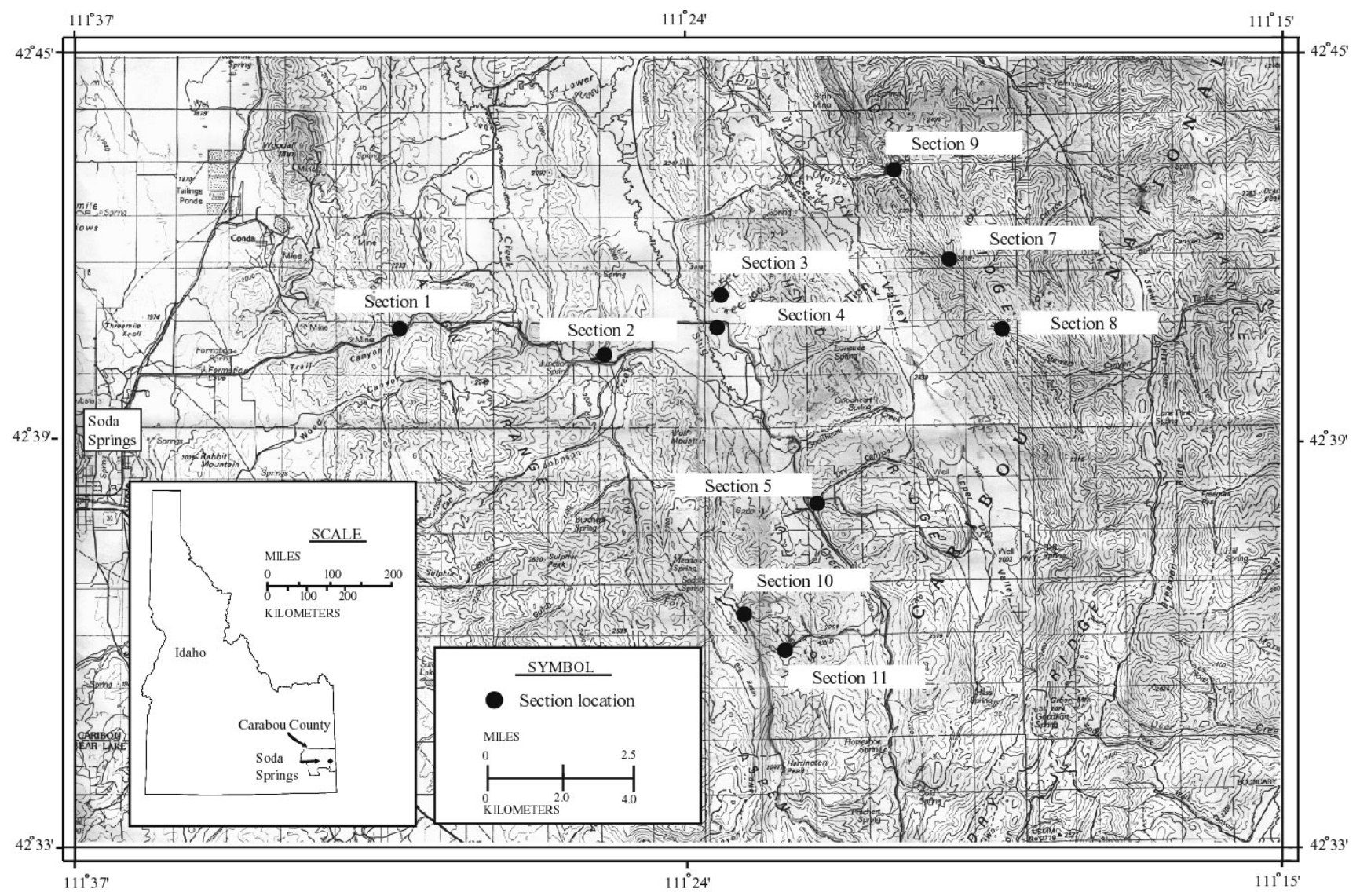

Figure 1. Index map of S. E. Idaho and location of sections of Rex
Chert (1-5, 7-11); section 1, 5, and 7 were measured and sampled

Table 1. GPS coordinates and elevations; both accurate within $<15$ meters $(49 \mathrm{ft})$

\begin{tabular}{lcll}
\hline $\begin{array}{c}\text { Section } \\
\text { Number }\end{array}$ & $\begin{array}{c}\text { Latitude } \\
(\mathbf{N})\end{array}$ & $\begin{array}{c}\text { Longitude } \\
(\mathbf{W})\end{array}$ & $\begin{array}{c}\text { Elevation } \\
(\mathbf{m})\end{array}$ \\
\hline Section 1 & $42^{\circ} 42.16^{\prime}$ & $111^{\circ} 29.07^{\prime}$ & 2122 \\
Section 2 & $42^{\circ} 41.78^{\prime}$ & $111^{\circ} 24.61^{\prime}$ & 1967 \\
Section 3 & $42^{\circ} 42.82^{\prime}$ & $111^{\circ} 21.93^{\prime}$ & 1974 \\
Section 4 & $42^{\circ} 42.17^{\prime}$ & $111^{\circ} 22.04^{\prime}$ & 1961 \\
Section 5 & $42^{\circ} 39.38^{\prime}$, & $111^{\circ} 19.76^{\prime}$ & 2042 \\
Section 5 & $42^{\circ} 39.39^{\prime}$ & $111^{\circ} 19.42^{\prime}$ & 2042 \\
Section 7 & $42^{\circ} 43.91^{\prime}$ & $111^{\circ} 17.37^{\prime}$ & 2248 \\
Section 8 & $42^{\circ} 42.15^{\prime 3}$ & $111^{\circ} 17.07^{\prime 3}$ & $2560^{3}$ \\
Section 9 & $42^{\circ} 44.85^{\prime}$, & $111^{\circ} 17.79^{\prime}$ & 2100 \\
Section 10 & $42^{\circ} 37.55^{\prime}$ & $111^{\circ} 21.13^{\prime}$ & 2314 \\
Section 11 & $42^{\circ} 36.89^{\prime}$ & $111^{\circ} 20.25^{\prime}$ & 2134 \\
\hline
\end{tabular}

${ }^{1} \mathrm{SW}$ end, ${ }^{2} \mathrm{NW}$ end, ${ }^{3}$ taken from topographic map 
the western phosphate industry in Idaho and provided a brief description of the mining history, ore occurrence, and geology, which has recently been updated by Lee (2001). McKelvey and others (1959) discussed in detail the Phosphoria Formation in the Western Phosphate Field. Cressman and Swanson (1964) provided detailed stratigraphy and petrology of these same rock units located in nearby SW Montana. Gulbrandsen and Krier (1980) discussed general aspects of the large and rich phosphate resources of the Phosphoria Formation in the vicinity of Soda Springs. Gulbrandsen $(1966,1975,1979)$ summarized bulk chemical compositions for various rock types of the phosphatic intervals of the Phosphoria Formation.

\section{Lithostratigraphy}

Three stratigraphic sections of the Rex Chert Member were measured and those three sections along with seven others were described in the field in SE Idaho (Fig. 1). Samples were collected from the three measured sections and their stratigraphic positions are indicated in Fig. 2.

The Phosphoria Formation in the Soda Springs area of SE Idaho consists of three members, which in ascending order are the Meade Peak Phosphatic Shale Member, the Rex Chert, and the informally named cherty shale (McKelvey and others, 1959; Montgomery and Cheney, 1967; Brittenham, 1976; Oberlindacher, 1990). The measured sections of this report focus on the Rex Chert in addition to the cherty shale member in one measured section. The Meade Peak unconformably overlies the Grandeur Tongue of the Permian Park City Formation, and the cherty shale member of the Phosphoria is overlain by the Triassic Dinwoody Formation.

The contacts between the Meade Peak and the Rex Chert and between the Rex Chert and the cherty shale member are gradational. The transitional rocks generally contain carbonates or carbonate-rich beds.

Section 1 was measured through a road cut that exposed the transition zone between the upper part of the Meade Peak and the Rex Chert and an adjacent quarry wall that exposed the cherty shale member. Section 5 was measured in a small quarry where the Rex Chert was being mined for surfacing roads. The underlying Meade Peak was not exposed and the overlying cherty shale was not present. Section 7 was measured along the exposed face of the now-abandoned South Maybe Canyon Mine and includes the upper Meade Peak rocks and transition rocks to the Rex Chert. Measurements record true thickness of strata.

In section 1, the Meade Peak Member is covered and the lowermost beds exposed include limestone alternating with black chert (Fig. 2, unit A). Most of the lower part of the Rex Chert is thin-bedded black and gray chert, which gives way up section to thickbedded massive gray chert (units B-F). Load casts are seen at the base of the thick chert. The uppermost massive chert beds are composed of composite beds displaying pinch-andswell structures (unit F). Above the uppermost massive thick chert bed are several thin, iron-rich, weathered chert beds (unit G). Sponge spicules are clearly seen in some of the chert beds, but thin sections reveal that most of the Rex Chert in this section is spicularite. The transition to the overlying cherty shale member is covered in the measured section, but in the outcrop on the opposite (south) side of the road, that position may be occupied by a chert-pebble conglomerate that is not seen on the north side of the road. The lower third of the cherty shale member consists of thin-bedded (up to $6 \mathrm{~cm}$ ) siliceous shale, with some beds displaying load casts (unit H1). Beds generally thicken up section. The middle third of the section consists of thin-bedded $(6-15 \mathrm{~cm})$ argillaceous chert with thinner $(<1-3 \mathrm{~mm})$ interbedded siliceous shale (unit H2). The upper third of the section is variable and consists of moderately thick-bedded $(10-30 \mathrm{~cm})$ argillaceous chert with sheared siliceous shale partings (unit H3). Near the top of the section occurs a body of pale-brown siliceous dolostone that appears to be the hinge of a fold and the remainder of the bed(?) is not exposed laterally from that body. This was probably originally a dolostone bed or laterally 


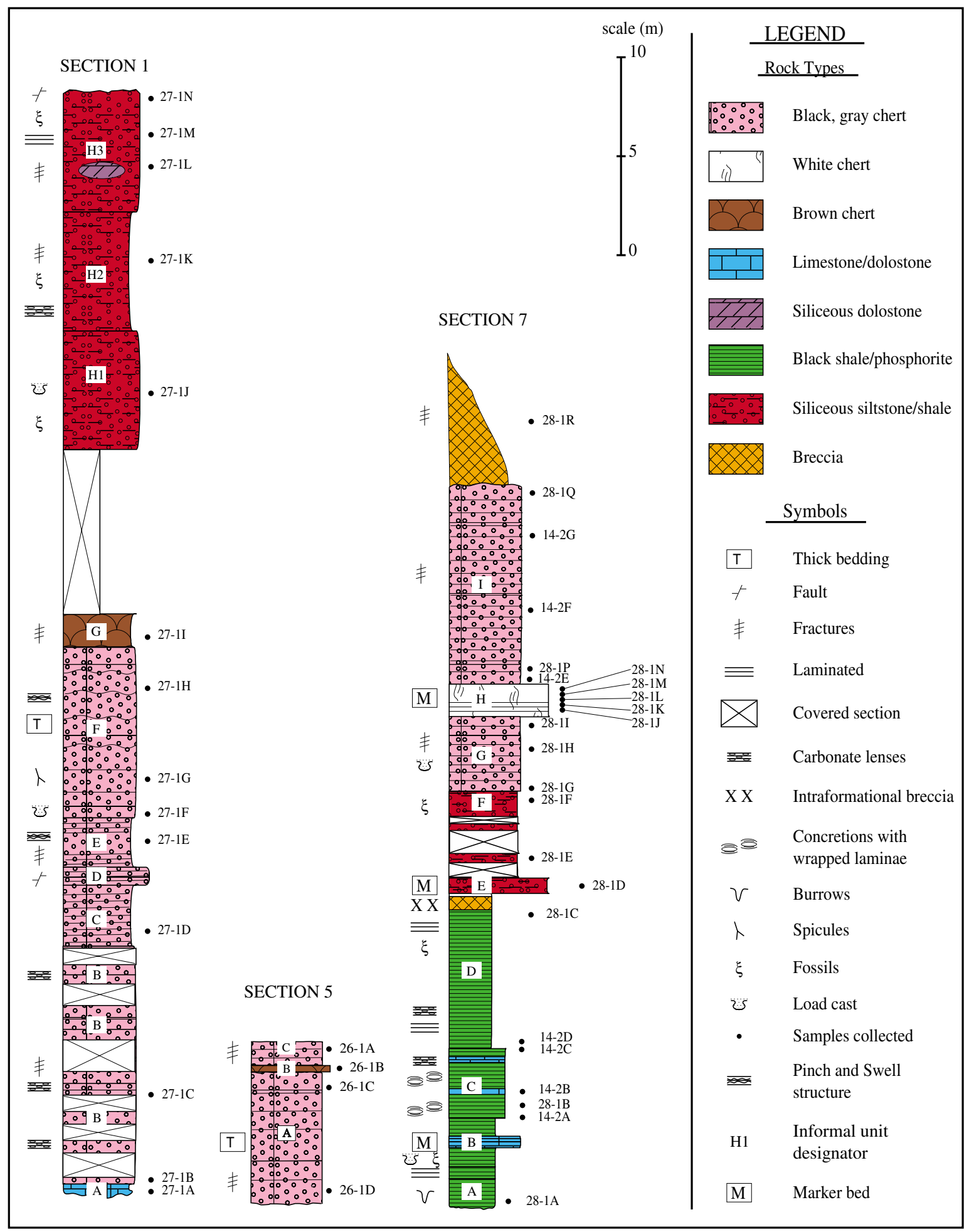

Figure 2. Measured and sampled sections of Rex Chert; units H1-H3 in section 1 is cherty shale member, units A-D in section 7 is Meade Peak Member 
extensive lens. A bed ( $40 \mathrm{~cm}$ thick) of laminated and sheared black siliceous shale occurs near the top of the section, which is capped by a nodular siliceous shale bed. The upper part of the section is highly sheared and is likely bounded by a fault. It is likely that the thickness of the Rex Chert and cherty shale members have been altered by folding and faulting. Bedding $\left(\mathrm{S}_{0}\right)$ in much of the Rex Chert has been obscured by development of generally low-relief stylolites $\left(\mathrm{S}_{1}\right)$ oriented subparallel to bedding. Locally, bedding has been transposed $\left(\mathrm{S}_{2}\right)$ so that it parallels axial planes of isoclinal folds that may have formed during compaction, or possibly during emplacement of the Paris thrust plate. $\mathrm{S}_{2}$ remained an active structural element as shown by quartz veins that formed perpendicular to $S_{2}$ and are offset along $\mathrm{S}_{2}$.

Section 5 was measured at its NW end in a quarry where the thickness of the Rex Chert is a minimum because the lower part of the section is covered and the upper part has been removed by quarrying. The Rex Chert consists of dark-gray, medium- to thick-bedded chert that is divided into two sections by an intervening friable brown layer of ferruginous chert (unit B) that has an earthy texture. This 30-40 cm-thick brown zone may be alteration along a fault or leaching of a chert bed. The cherts in this section are spicularites, as determined from thin sections. The chert shows bedding-parallel pressure-solution cleavage as indicated by truncated microfossils and accumulations of relatively insoluble components such as sericite and hematite.

In section 7, the upper part of the Meade Peak Member (units A-D) and the transition to the Rex Chert (units E-F) are well exposed. Alternating beds of black shale, phosphorite, and carbonate characterize the Meade Peak exposure. Carbonates (calcite and dolomite) occur as beds, horizons of disconnected concretions, and as isolated concretions in some shale beds. Generally the carbonates thicken up section from nodules to thin beds to thicker beds, except near the base of the exposed section where a $0.6 \mathrm{~m}$ thick carbonate "marker bed" occurs. These marker beds (see below also) crop out over a distance of about 3 miles $(4.8 \mathrm{~km})$ in the North, Middle, and South Maybe Canyon mines in the Dry Ridge Mountains. Shale and phosphorite laminae wrap around the carbonate concretions indicating an early diagenetic origin (pre-compaction). The Meade Peak is overlain by a $0.8 \mathrm{~m}$ thick marker bed of siliceous siltstone (Fig. 2, unit E). That bed is overlain by a section that is partly covered, but consists predominantly of thin-bedded siliceous siltstone (unit F). That transition zone of siliceous siltstones is overlain by thin- to medium-bedded $(5-30 \mathrm{~cm})$ black chert with brown upper and lower margins on each bed (unit G). Load casts are seen at the base of some beds. A few thin siliceous siltstone beds also occur in unit $\mathrm{G}$. Those cherts are overlain by $1 \mathrm{~m}$ of thin-bedded white chert that is overlain by a 1 $\mathrm{m}$ thick white chert marker bed (unit $\mathrm{H}$ ). The white cherts are overlain by $10 \mathrm{~m}$ of thin- to medium-bedded (to $30 \mathrm{~cm}$ ) black and gray chert (unit I) that is very similar to cherts in unit $\mathrm{G}$. The section is capped by a $8 \mathrm{~m}$ thick, discontinuous chert megabreccia with a calcitebearing quartz cement. This breccia body may be a slump deposit or fault-zone deposit.

\section{METHODS}

\section{Field Sampling}

The samples within the measured sections obtained for analyses were collected as individual beds, where possible. In several places, where beds were too thick to collect in their entirety, portions of beds were collected. This approach provides an opportunity to determine the changes in composition of like samples through the history of deposition of stratigraphic sections. The choice of beds sampled is intended to provide uniform representation of each section. In addition, unusual rock types were sampled, for example 
a large dolomitic body in the cherty shale member. About $0.1-1 \mathrm{~kg}$ of rock was collected for each bed.

\section{Rock Sample Preparation}

A representative slab was cut through the entire thickness of each chert bed sample and was crushed in a mechanical jaw crusher, and then powdered in a roller mill. An aliquot of the powdered material was analyzed by X-ray diffraction for mineral content and a second aliquot was sent to a contract laboratory for chemical analyses. All splits were obtained with a riffle splitter to ensure similarity with the whole sample. Splits of about $15-50 \mathrm{~g}$ were sent to the contract laboratory and the remaining powders for all samples are archived at the USGS. A second slab of each chert bed sample was cut into one or more thin sections for petrographic analysis.

\section{Analyses}

Samples were analyzed for 40 major, minor, and trace elements using acid digestion in conjunction with inductively coupled plasma-atomic emission spectrometry (ICP-AES). For the 40-element analysis (referred to as ICP-40), a split was dissolved using a lowtemperature $\left(<150^{\circ} \mathrm{C}\right)$ digestion with concentrated hydrochloric, hydrofluoric, nitric, and perchloric acids (Jackson and others, 1987). The analytical contractor modified this procedure to shorten the digestion time (P. Lamothe, USGS, oral communication). The acidic sample solutions were taken to dryness and the residue was dissolved with $1 \mathrm{ml}$ of aqua regia and then diluted to $10.0 \mathrm{~g}$ with $1 \%$ (volume/volume) nitric acid. Strontium and Ba concentrations were determined by both ICP-40 and ICP-16 (see below) techniques and the two data sets are comparable $\left(\mathrm{R}^{2}=0.999\right.$ for $\mathrm{Sr}$ and 0.997 for $\left.\mathrm{Ba}\right)$ and are reported in Table 3. Manganese concentrations were also provided by both ICP techniques and they have comparable accuracy and precision, however, the ICP-40 data set is the only one reported because it has a much lower detection limit, 4 parts per million (ppm) compared to $100 \mathrm{ppm}$ for ICP-16. The ICP-40 technique measures the following elements above the indicated ppm limits: Au 8 ppm, Bi 50 ppm, Sn 50 ppm, Ta 40 ppm, and U 100 ppm; however, no samples had concentrations above those quantification limits and those elements are not included in Table 3.

Another split of the each sample was fused with lithium metaborate then analyzed by ICP-AES after acid dissolution of the fusion mixture. This technique, referred to as ICP16 , provides analysis of all major elements, including $\mathrm{Si}$, and a few minor and trace elements. Cherts are very high silica rocks yet the accuracy of Si determinations is quite good, probably about $2-4 \%$ based on the total-oxide sums. Si measurement is not possible using the 4-acid digestion ICP-40 technique because it is lost as a volatile fluoride compound during digestion. Analysis of major elements using the fusion technique also provides a compositional check on the concentrations of these same elements as measured by acid digestion. Titanium and $\mathrm{Cr}$ were analyzed using both ICP techniques, but only data from the ICP 16 technique are given in Table 3 because the fusion technique more completely digests resistant minerals that might contain those elements.

Selenium concentrations were determined using hydride generation followed by atomic absorption (AA) spectrometry. Selenium is not reported using either of the ICP techniques, as it is generally volatilized during sample preparation. The hydride generation combined with AA technique was also used to determine concentrations of As, Sb, and Tl. The hydride analytical technique is considered to be more sensitive than the acid digestion ICP-AES analytical technique for As and are the data reported here. Mercury was determined by cold vapor atomic absorption spectrometry.

Total $\mathrm{S}$ and total $\mathrm{C}$ were measured using combustion in oxygen followed by infrared measurement of the evolved gas. For the other forms of carbon, carbonate carbon was 
Table 3. Chemical composition of rocks from three sections (1, 5, 7; Figs. 1, 2) of Rex Chert and adjacent rocks; samples are listed in stratigraphic order; major oxides, $\mathrm{C}$, and $\mathrm{S}$ in wt.\%, others in ppm

\begin{tabular}{|c|c|c|c|c|c|c|c|c|c|c|c|c|}
\hline Lab No. & Sample No. & Sample Description & Lithology & $\begin{array}{c}\mathrm{SiO} 2 \\
\mathrm{ICP}-16\end{array}$ & $\begin{array}{l}\mathrm{A} 12 \mathrm{O} 3 \\
\mathrm{ICP}-16\end{array}$ & $\begin{array}{l}\mathrm{Fe} 2 \mathrm{O} 3 \\
\mathrm{ICP}-16\end{array}$ & $\begin{array}{c}\mathrm{TiO} 2 \\
\text { ICP-16 }\end{array}$ & $\begin{array}{c}\mathrm{CaO} \\
\mathrm{ICP}-16\end{array}$ & $\begin{array}{c}\mathrm{K} 2 \mathrm{O} \\
\mathrm{ICP}-16\end{array}$ & $\begin{array}{c}\mathrm{MgO} \\
\text { ICP-16 }\end{array}$ & $\begin{array}{l}\mathrm{Na} 2 \mathrm{O} \\
\mathrm{ICP}-16\end{array}$ & $\begin{array}{c}\mathrm{P} 2 \mathrm{O} 5 \\
\mathrm{ICP}-16\end{array}$ \\
\hline \multicolumn{13}{|c|}{ SECTION 1: REX CHERT 1A-1I; CHERTY SHALE MEMBER 1J-1N } \\
\hline C-197024 & $601-27-1 \mathrm{~N}$ & Brown, calcareous & Cherty-calcareous shale bed & 87.5 & 3.27 & 0.77 & 0.22 & 2.28 & 0.61 & 1.09 & 0.63 & 0.48 \\
\hline C-197023 & $601-27-1 \mathrm{M}$ & Black & Laminated siliceous shale & 78.7 & 8.41 & 2.66 & 0.55 & 1 & 1.95 & 0.8 & 0.59 & 0.7 \\
\hline C-197021 & $601-27-1 \mathrm{~L} 1$ & Brown, earthy, porous & Argillaceous chert bed & 92.6 & 2.74 & 1.92 & 0.17 & 0.43 & 0.3 & 0.18 & 0.61 & 0.32 \\
\hline C-197020 & $601-27-1 \mathrm{~K} 2$ & Brown, calcareous & Argillaceous chert bed & 92 & 2.4 & 0.64 & 0.17 & 1.41 & 0.43 & 0.73 & 0.31 & 0.2 \\
\hline C-197019 & $601-27-1 \mathrm{~K}$ & Brown & Shaly interbed & 90.9 & 3.48 & 0.96 & 0.23 & 0.8 & 0.64 & 0.32 & 0.42 & 0.44 \\
\hline C-197018 & $601-27-1 \mathrm{~J}$ & Brown, calcareous & Cherty shale bed & 86.2 & 3.82 & 1.16 & 0.23 & 2.01 & 0.78 & 0.95 & 0.49 & 0.44 \\
\hline C-197017 & $601-27-1 \mathrm{I}$ & Brown, Fe stained & Phosphatic-ferruginous chert bed & 83.2 & 2.55 & 4.68 & 0.12 & 3.69 & 0.64 & 0.45 & 0.08 & 3.05 \\
\hline C-197015 & $601-27-1 \mathrm{G}$ & Gray, spicular & Base of massive thick chert bed & 95.6 & 1.19 & 1.4 & 0.03 & 0.97 & 0.22 & 0.12 & 0.03 & 0.85 \\
\hline C-197014 & $601-27-1 \mathrm{~F}$ & Gray & Chert bed & 99.9 & 0.26 & 0.23 & $<0.02$ & 0.39 & 0.02 & $<0.02$ & 0.01 & 0.28 \\
\hline C-197013 & $601-27-1 \mathrm{E}$ & Gray & Chert bed & 96 & 0.62 & 0.44 & $<0.02$ & 1.27 & 0.08 & 0.05 & 0.03 & 0.96 \\
\hline C-197012 & 601-27-1D & Black & Chert bed & 98.2 & 0.6 & 0.33 & 0.02 & 0.21 & 0.1 & 0.05 & 0.04 & 0.09 \\
\hline C-197011 & $601-27-1 \mathrm{C}$ & Black, calcareous & Chert bed & 97.5 & 0.43 & 0.19 & 0.02 & 1.1 & 0.06 & 0.18 & 0.04 & 0.16 \\
\hline C-197010 & $601-27-1 B$ & Black, calcareous & Chert bed & 93.5 & 0.59 & 0.17 & 0.03 & 2.31 & 0.07 & 0.93 & 0.05 & 0.28 \\
\hline C-197009 & $601-27-1 \mathrm{~A}$ & Gray & Siliceous limestone bed & 44.3 & 0.2 & 0.06 & $<0.02$ & 30.9 & 0.04 & 0.48 & 0.04 & 0.05 \\
\hline \multicolumn{13}{|c|}{ SECTION 5} \\
\hline C-197005 & $601-26-1 \mathrm{~A}$ & Dark gray, fractured & Chert bed & 98.6 & 0.55 & 0.31 & 0.02 & 0.22 & 0.07 & 0.05 & 0.04 & 0.14 \\
\hline C-197006 & $601-26-1 \mathrm{~B}$ & Earthy, brown & Leached zone in chert & 93.9 & 1.49 & 2.65 & 0.08 & 0.43 & 0.17 & 0.12 & 0.04 & 0.3 \\
\hline \multicolumn{13}{|c|}{ SECTION 7: MEADE PEAK 601-28-1A-1C: REX CHERT 601-28-1D THROUGH 601-28-1R1 } \\
\hline $\mathrm{C}-197045$ & $601-28-1 \mathrm{R} 1$ & Pale brown-gray chert clast & Breccia & 99.5 & 0.4 & 0.07 & $<0.02$ & 1.05 & 0.05 & 0.55 & 0.05 & 0.14 \\
\hline C-197044 & $601-28-1 Q$ & Dark gray, fractured & Chert bed & 101 & 0.4 & 0.06 & $<0.02$ & 0.46 & 0.04 & 0.02 & 0.05 & 0.28 \\
\hline C-205123 & $502-14-2 \mathrm{G}$ & Gray & Chert bed & 95.1 & 0.55 & 0.11 & 0.03 & 0.41 & 0.05 & 0.02 & 0.08 & 0.25 \\
\hline C- 205122 & $502-14-2 \mathrm{~F}$ & Black, fractured & Chert bed & 95.81 & 0.6 & 0.11 & 0.03 & 0.43 & 0.06 & 0.03 & 0.08 & 0.25 \\
\hline C-205121 & $502-14-2 \mathrm{E}$ & Brown & Chert bed & 94.29 & 0.72 & 0.21 & 0.03 & 0.49 & 0.08 & 0.03 & 0.07 & 0.3 \\
\hline C-197043 & $601-28-1 \mathrm{P}$ & Gray, mottled & Chert bed & 96.7 & 0.25 & 0.04 & $<0.02$ & 2.92 & 0.01 & 0.7 & 0.04 & 0.09 \\
\hline C- 197042 & $601-28-1 \mathrm{~N}$ & White, upper $15 \mathrm{~cm}$ margin & $1 \mathrm{~N}-1 \mathrm{~K}=$ parts of a $80 \mathrm{~cm}$ thick & 102 & 0.28 & 0.17 & $<0.02$ & 0.3 & 0.04 & 0.02 & 0.04 & 0.18 \\
\hline C-197041 & $601-28-1 \mathrm{M}$ & White, middle $7 \mathrm{~cm}$ & white chert bed & 101 & 0.32 & 0.04 & $<0.02$ & 0.18 & 0.01 & 0.02 & 0.03 & 0.09 \\
\hline C- 197040 & $601-28-1 \mathrm{~L}$ & White, $7-15 \mathrm{~cm}$ above base & chert & 101 & 0.23 & 0.13 & $<0.02$ & 0.27 & $<0.01$ & 0.02 & 0.03 & 0.16 \\
\hline C-197039 & $601-28-1 \mathrm{~K}$ & White, lower margin $(8 \mathrm{~cm})$ & chert & 101 & 0.36 & 0.1 & $<0.02$ & 0.35 & 0.02 & 0.02 & 0.03 & 0.2 \\
\hline C-197036 & $601-28-1 J 1$ & Pale brown, upper bed margin & Chert & 100 & 0.28 & 0.1 & $<0.02$ & 0.3 & 0.02 & 0.02 & 0.04 & 0.18 \\
\hline C-197037 & $601-28-1 \mathrm{~J} 2$ & White-gray, main bed, dense & Chert & 101 & 0.32 & 0.04 & $<0.02$ & 0.2 & 0.02 & $<0.02$ & 0.03 & 0.11 \\
\hline C- 197038 & $601-28-1 \mathrm{~J} 3$ & White-gray, lower bed margin & Chert & 101 & 0.42 & 0.09 & $<0.02$ & 0.34 & 0.02 & 0.02 & 0.03 & 0.21 \\
\hline C-197035 & $601-28-1 \mathrm{I}$ & Black-brown & Chert bed & 96.7 & 0.77 & 0.21 & 0.03 & 0.5 & 0.1 & 0.03 & 0.08 & 0.32 \\
\hline C-197034 & $601-28-1 \mathrm{H}$ & Pale brown, silty & Chert bed & 99.5 & 0.43 & 0.17 & 0.02 & 0.34 & 0.05 & 0.03 & 0.07 & 0.2 \\
\hline C-197031 & $601-28-1 \mathrm{G} 1$ & Pale brown, upper bed margin & Chert & 96 & 1.38 & 0.37 & 0.05 & 1.01 & 0.22 & 0.08 & 0.12 & 0.7 \\
\hline C-197032 & $601-28-1 \mathrm{G} 2$ & Brown, main bed & Chert & 90.7 & 0.93 & 0.26 & 0.05 & 0.67 & 0.14 & 0.05 & 0.11 & 0.39 \\
\hline C-197033 & $601-28-1 \mathrm{G} 3$ & Pale brown, lower bed margin & Chert & 95.4 & 1.64 & 0.6 & 0.08 & 1.48 & 0.27 & 0.12 & 0.12 & 1.01 \\
\hline
\end{tabular}


Table 3 continued

\begin{tabular}{|c|c|c|c|c|c|c|c|c|c|c|c|c|c|c|c|c|}
\hline Lab No. & Sample No. & \multicolumn{2}{|c|}{ Sample Description } & \multicolumn{3}{|c|}{ Lithology } & $\begin{array}{c}\mathrm{SiO} 2 \\
\mathrm{ICP}-16\end{array}$ & $\begin{array}{l}\mathrm{A} 12 \mathrm{O} 3 \\
\mathrm{ICP}-16\end{array}$ & $\begin{array}{l}\mathrm{Fe} 2 \mathrm{O} 3 \\
\mathrm{ICP}-16\end{array}$ & $\begin{array}{c}\mathrm{TiO} 2 \\
\mathrm{ICP}-16\end{array}$ & $\begin{array}{c}\mathrm{CaO} \\
\mathrm{ICP}-16\end{array}$ & $\begin{array}{l}\mathrm{K} 2 \mathrm{O} \\
\mathrm{ICP}-16\end{array}$ & $\begin{array}{l}\mathrm{MgO} \\
\mathrm{ICP}-16\end{array}$ & $\begin{array}{l}\mathrm{Na2O} \\
\mathrm{ICP}-16\end{array}$ & $\begin{array}{l}\mathrm{P} 2 \mathrm{O} 5 \\
\mathrm{ICP}-16\end{array}$ & \\
\hline $\mathrm{C}-197030$ & $601-28-1 \mathrm{~F}$ & \multicolumn{2}{|c|}{ Gray-brown, fossiliferous } & \multicolumn{3}{|c|}{ Phosphatic cherty shale bed } & 89 & 3.48 & 1.24 & 0.18 & 2.46 & 0.69 & 0.3 & 0.15 & 1.72 & \\
\hline C-197029 & $601-28-1 \mathrm{E}$ & \multirow{2}{*}{\multicolumn{2}{|c|}{$\begin{array}{l}\text { Black, thin-bedded } \\
\text { Gray-brown, mid. Thick bed }\end{array}$}} & \multicolumn{3}{|c|}{ Siliceous siltstone } & 75 & 10.7 & 2.09 & 0.78 & 0.17 & 2.58 & 0.65 & 1.2 & 0.09 & \\
\hline C-197028 & $601-28-1 \mathrm{D}$ & & & \multicolumn{3}{|c|}{ Siliceous siltstone, marker bed } & 78.5 & 11.1 & 0.96 & 0.82 & 0.34 & 2.57 & 0.61 & 1.32 & 0.05 & \\
\hline C-197027 & $601-28-1 \mathrm{C}$ & \multicolumn{2}{|c|}{ Black } & \multicolumn{3}{|c|}{ Phosphatic-calcareous shale } & 48.3 & 8.35 & 2.75 & 0.48 & 17.1 & 1.88 & 0.7 & 0.66 & 5.5 & \\
\hline C-205120 & $502-14-2 \mathrm{D}$ & \multicolumn{2}{|c|}{ Black, carbonaceous } & \multicolumn{3}{|c|}{ Phosphorite } & 3.96 & 0.59 & 0.13 & 0.03 & 46.8 & 0.12 & 0.07 & 0.16 & 33.1 & \\
\hline C-205119 & $502-14-2 \mathrm{C}$ & \multicolumn{2}{|c|}{ Black nodule } & \multicolumn{3}{|c|}{ Carbonate nodule in black shale } & 3.53 & 0.47 & 0.16 & 0.03 & 47.8 & 0.08 & 2.74 & 0.22 & 2.82 & \\
\hline C-205118 & $502-14-2 B$ & \multicolumn{2}{|c|}{ Black, carbonaceous } & \multicolumn{3}{|c|}{ Phosphatic shale } & 16.1 & 2.48 & 0.81 & 0.13 & 35.1 & 0.65 & 0.91 & 0.63 & 15.4 & \\
\hline C-197026 & $601-28-1 B$ & \multicolumn{2}{|c|}{ Black nodule } & & late nodule in & lack shale & 1.1 & 0.15 & 0.06 & $<0.020$ & 48.7 & 0.01 & 3.3 & 0.11 & 2.8 & \\
\hline C-205117 & $502-14-2 \mathrm{~A}$ & \multicolumn{2}{|c|}{ Black, carbonaceous } & Phos & latic shale & & 23.8 & 3.87 & 1.3 & 0.23 & 31.5 & 0.96 & 1.09 & 0.74 & 9.56 & \\
\hline C-197025 & $601-28-1 \mathrm{~A}$ & Black, car & aceous & Phos & tatic shale & & 46 & 7.29 & 2.37 & 0.48 & 11.1 & 1.94 & 1.44 & 1.2 & 4.42 & \\
\hline $\begin{array}{l}\text { Sample No. } \\
\text { Section } 1\end{array}$ & $\Sigma \mathrm{CO} 2$ & Total & CT & $\mathrm{CC}$ & Corg. & ST & $\mathrm{Ag}$ & As & $\mathrm{B}$ & & $\mathrm{Ba}$ & $\mathrm{Be}$ & $\mathrm{Cd}$ & & $\mathrm{Ce}$ & $\mathrm{Co}$ \\
\hline$\underline{\text { Section } 1}$ & Acid. & -- & Comb. & Acid. & Difference & Comb. & ICP-40 & Hydride & ICP & & ICP-16 & ICP-40 & ICP-40 & & ICP-40 & ICP-40 \\
\hline $601-27-1 \mathrm{~N}$ & 1.69 & 98.6 & 1.32 & 0.46 & 0.86 & 0.2 & $<2$ & 3.1 & 59 & & 611 & $<1$ & $<2$ & & 19 & $<2$ \\
\hline $601-27-1 \mathrm{M}$ & 0.03 & 95.4 & 1.81 & 0.01 & 1.8 & 0.05 & $<2$ & 12.6 & 24 & & 243 & 2 & $<2$ & & 36 & 3 \\
\hline $601-27-1 \mathrm{~L} 2$ & 23.4 & 101 & 6.36 & 6.39 & -0.03 & $<0.05$ & $<2$ & 1.1 & 2 & & 27 & $<1$ & $<2$ & & 6 & $<2$ \\
\hline 601-27-1L1 & 0.02 & 99.3 & 0.11 & 0.01 & 0.1 & $<0.05$ & $<2$ & 3.8 & 5 & & 60 & $<1$ & $<2$ & & 12 & $<2$ \\
\hline $601-27-1 \mathrm{~K} 2$ & 1.15 & 99.5 & 0.77 & 0.31 & 0.46 & 0.12 & $<2$ & 2.5 & 48 & & 439 & $<1$ & $<2$ & & 13 & $<2$ \\
\hline $601-27-1 \mathrm{~K}$ & 0.23 & 98.5 & 0.76 & 0.06 & 0.7 & $<0.05$ & $<2$ & 3.8 & 13 & & 125 & $<1$ & $<2$ & & 19 & 2 \\
\hline $601-27-1 \mathrm{~J}$ & 1.58 & 97.7 & 1.57 & 0.43 & 1.14 & 0.07 & $<2$ & 3.1 & 12 & & 124 & $<1$ & $<2$ & & 20 & 3 \\
\hline 601-27-1I & 0.08 & 98.5 & 0.31 & 0.02 & 0.29 & $<0.05$ & $<2$ & 13.2 & 7 & & 81 & 2 & $<2$ & & 87 & $<2$ \\
\hline $601-27-1 \mathrm{H}$ & 0.02 & 100 & 0.13 & 0.01 & 0.12 & $<0.05$ & $<2$ & 6.1 & 8 & & 80 & $<1$ & $<2$ & & 9 & $<2$ \\
\hline $601-27-1 \mathrm{G}$ & 0.02 & 100 & 0.13 & 0.01 & 0.12 & $<0.05$ & $<2$ & 5.1 & 3 & & 48 & $<1$ & $<2$ & & 10 & $<2$ \\
\hline $601-27-1 F$ & 0.01 & 101 & 0.01 & $<0.003$ & 0.007 & $<0.05$ & $<2$ & 0.9 & 1 & & 16 & $<1$ & $<2$ & & $<5$ & $<2$ \\
\hline $601-27-1 E$ & 0.02 & 99.7 & 0.27 & 0.01 & 0.26 & $<0.05$ & $<2$ & 2.5 & 3 & & 30 & $<1$ & $<2$ & & $<5$ & $<2$ \\
\hline 601-27-1D & 0.03 & 99.7 & 0.39 & 0.01 & 0.38 & $<0.05$ & $<2$ & 1.3 & 5 & & 49 & $<1$ & $<2$ & & $<5$ & $<2$ \\
\hline $601-27-1 \mathrm{C}$ & 0.79 & 100 & 0.38 & 0.22 & 0.16 & $<0.05$ & $<2$ & 0.7 & 2 & & 27 & $<1$ & $<2$ & & $<5$ & $<2$ \\
\hline 601-27-1B & 2.2 & 100 & 0.91 & 0.59 & 0.32 & $<0.05$ & $<2$ & 1 & 2 & & 24 & $<1$ & $<2$ & & $<5$ & $<2$ \\
\hline $601-27-1 \mathrm{~A}$ & 25.1 & 101 & 7.06 & 6.85 & 0.21 & $<0.05$ & $<2$ & $<0.6$ & $\varepsilon$ & & $<10$ & $<1$ & $<2$ & & $<5$ & $<2$ \\
\hline$\underline{\text { Section } 5}$ & & & & & & & & & & & & & & & & \\
\hline 601-26-1A & 0.02 & 100 & 0.09 & 0.01 & 0.08 & $<0.05$ & $<2$ & 1.6 & 27 & & 261 & $<1$ & $<2$ & & $<5$ & $<2$ \\
\hline 601-26-1B & 0.01 & 99.2 & 0.11 & $<0.003$ & 0.107 & $<0.05$ & $<2$ & 14.5 & 8 & & 84 & $<1$ & $<2$ & & 8 & $<2$ \\
\hline $601-26-1 \mathrm{C}$ & 0.03 & 99.9 & 0.13 & 0.01 & 0.12 & $<0.05$ & $<2$ & 2.7 & 12 & & 115 & $<1$ & $<2$ & & 5 & $<2$ \\
\hline 601-26-1D & 0.01 & 99.9 & 0.19 & $<0.003$ & 0.187 & 0.05 & $<2$ & 2.4 & 42 & & 415 & $<1$ & $<2$ & & 6 & $<2$ \\
\hline Section 7 & & & & & & & & & & & & & & & & \\
\hline 601-28-1R1 & 1.1 & 102 & 0.38 & 0.3 & 0.08 & $<0.05$ & $<2$ & 4.3 & 2 & & 32 & $<1$ & $<2$ & & $<5$ & $<2$ \\
\hline $601-28-1 Q$ & 0.02 & 102 & 0.11 & 0.01 & 0.1 & $<0.05$ & $<2$ & 5.4 & 2 & & 26 & $<1$ & $<2$ & & $<5$ & $<2$ \\
\hline $502-14-2 \mathrm{G}$ & 0.03 & 96.6 & 0.09 & 0.01 & 0.08 & $<0.05$ & $<2$ & 3.7 & 3 & & 35 & $<1$ & $<2$ & & $<5$ & $<2$ \\
\hline $502-14-2 \mathrm{~F}$ & 0.02 & 97.4 & 0.17 & 0.01 & 0.16 & $<0.05$ & $<2$ & 1.1 & 3 & & 37 & $<1$ & $<2$ & & $<5$ & $<2$ \\
\hline $502-14-2 \mathrm{E}$ & 0.02 & 96.3 & 0.05 & 0.01 & 0.04 & $<0.05$ & $<2$ & 2.2 & 6 & & 64 & $<1$ & $<2$ & & $<5$ & $<2$ \\
\hline $601-28-1 P$ & 2.78 & 103 & 0.81 & 0.76 & 0.05 & $<0.05$ & $<2$ & $<0.6$ & 1 & & 23 & $<1$ & $<2$ & & $<5$ & $<2$ \\
\hline $601-28-1 \mathrm{~N}$ & 0.01 & 103 & 0.02 & $<0.003$ & 0.017 & $<0.05$ & $<2$ & 0.9 & 2 & & 28 & $<1$ & $<2$ & & $<5$ & $<2$ \\
\hline $601-28-1 \mathrm{M}$ & 0.01 & 102 & 0.01 & $<0.003$ & 0.007 & $<0.05$ & $<2$ & $<0.6$ & 2 & & 26 & $<1$ & $<2$ & & $<5$ & $<2$ \\
\hline
\end{tabular}


Table 3 continued

\begin{tabular}{|c|c|c|c|c|c|c|c|c|c|c|c|c|c|c|c|}
\hline $\begin{array}{l}\text { Sample No. } \\
\text { Section } 7 \\
\end{array}$ & $\begin{array}{l}\mathrm{CO} 2 \\
\text { Acid. }\end{array}$ & $\begin{array}{c}\text { Total } \\
--\end{array}$ & $\begin{array}{c}\text { CT } \\
\text { Comb. }\end{array}$ & $\begin{array}{c}\mathrm{CC} \\
\text { Acid. }\end{array}$ & \multicolumn{2}{|c|}{$\begin{array}{c}\text { Corg. } \\
\text { Difference }\end{array}$} & $\begin{array}{c}\text { ST } \\
\text { Comb. }\end{array}$ & $\begin{array}{c}\mathrm{Ag} \\
\mathrm{ICP}-40\end{array}$ & $\begin{array}{c}\text { As } \\
\text { Hydride }\end{array}$ & $\begin{array}{c}\mathrm{Ba} \\
\mathrm{ICP}-40\end{array}$ & $\begin{array}{c}\mathrm{Ba} \\
\text { ICP-16 }\end{array}$ & $\begin{array}{c}\mathrm{Be} \\
\mathrm{ICP}-40\end{array}$ & $\begin{array}{c}\mathrm{Cd} \\
\mathrm{ICP}-40\end{array}$ & $\begin{array}{c}\mathrm{Ce} \\
\text { ICP-40 }\end{array}$ & $\begin{array}{c}\text { Co } \\
\text { ICP-40 }\end{array}$ \\
\hline $601-28-1 \mathrm{~L}$ & 0.01 & 102 & 0.03 & $<0.003$ & & & $<0.05$ & $<2$ & 2.8 & 21 & 22 & $<1$ & $<2$ & $<5$ & $<2$ \\
\hline $601-28-1 \mathrm{~K}$ & 0.02 & 102 & 0.02 & 0.01 & & & $<0.05$ & $<2$ & 0.9 & 25 & 33 & $<1$ & 2 & $<5$ & $<2$ \\
\hline $601-28-1 \mathrm{~J} 1$ & $<0.01$ & 101 & 0.03 & $<0.003$ & & & $<0.05$ & $<2$ & 2.3 & 35 & 37 & $<1$ & 2 & $<5$ & $<2$ \\
\hline $601-28-1 \mathrm{~J} 2$ & $<0.01$ & 102 & 0.02 & $<0.003$ & & & $<0.05$ & $<2$ & 0.8 & 39 & 44 & $<1$ & $<2$ & $<5$ & $<2$ \\
\hline $601-28-1 \mathrm{~J} 3$ & 0.01 & 102 & 0.03 & $<0.003$ & & & $<0.05$ & 7 & 1.2 & 37 & 40 & $<1$ & $<2$ & $<5$ & $<2$ \\
\hline 601-28-1I & 0.02 & 98.8 & 0.15 & 0.01 & & & $<0.05$ & $<2$ & 2.1 & 43 & 43 & $<1$ & $<2$ & $<5$ & $<2$ \\
\hline $601-28-1 \mathrm{H}$ & 0.02 & 101 & 0.12 & 0.01 & & & $<0.05$ & $<2$ & 0.6 & 29 & 33 & $<1$ & $<2$ & 8 & $<2$ \\
\hline $601-28-1 \mathrm{G} 1$ & 0.05 & 100 & 0.18 & 0.01 & & & $<0.05$ & 3 & 6.6 & 51 & 50 & $<1$ & 4 & 7 & $<2$ \\
\hline $601-28-1 \mathrm{G} 2$ & 0.02 & 93.3 & 0.35 & 0.01 & & & 0.05 & $<2$ & 4.1 & 62 & 56 & $<1$ & 4 & 5 & $<2$ \\
\hline $601-28-1 \mathrm{G} 3$ & 0.07 & 101 & 0.21 & 0.02 & & & $<0.05$ & 3 & 11 & 62 & 59 & $<1$ & 11 & 10 & $<2$ \\
\hline $601-28-1 \mathrm{~F}$ & 0.11 & 99.3 & 0.18 & 0.03 & & & $<0.05$ & 3 & 16.8 & 108 & 108 & 1 & 22 & 19 & $<2$ \\
\hline $601-28-1 \mathrm{E}$ & $<0.01$ & 93.1 & 3.35 & $<0.003$ & & & 0.42 & $<2$ & 14.2 & 342 & 336 & 1 & $<2$ & 47 & $<2$ \\
\hline 601-28-1D & $<0.01$ & 96.3 & 0.76 & $<0.003$ & & & 0.18 & 6 & 1.9 & 365 & 351 & 1 & 7 & 46 & $<2$ \\
\hline $601-28-1 C$ & 8.18 & 93.9 & 4.62 & 2.23 & & & 0.29 & $<2$ & 23.2 & 367 & 346 & 1 & 5 & 62 & 8 \\
\hline $502-14-2 \mathrm{D}$ & 1.45 & 86.4 & 8.84 & 0.4 & & & 1.08 & $<2$ & 3.8 & 56 & 49 & 1 & 79 & 21 & $<2$ \\
\hline $502-14-2 \mathrm{C} 1$ & 38.7 & 96.6 & 12.1 & 10.6 & & & 0.28 & $<2$ & 2.5 & 22 & 17 & $<1$ & 22 & 6 & $<2$ \\
\hline $502-14-2 B$ & 12.3 & 84.5 & 13.8 & 3.36 & & & 1.86 & 8 & 26.7 & 88 & 91 & 1 & 35 & 29 & 3 \\
\hline $601-28-1 B$ & 39.3 & 95.4 & 13.8 & 10.7 & & & 0.27 & $<2$ & 3.3 & 14 & 11 & $<1$ & 15 & $<5$ & $<2$ \\
\hline $502-14-2 \mathrm{~A}$ & 12.8 & 85.8 & 15.7 & 3.49 & & & 2.52 & 14 & 42.5 & 108 & 106 & 2 & 194 & 27 & 2 \\
\hline $601-28-1 A$ & 5.49 & 81.7 & 13.3 & 1.5 & & & 2.97 & 15 & 32.2 & 223 & 212 & 2 & 37 & 52 & 4 \\
\hline $\begin{array}{l}\text { Sample No. } \\
\text { Section } 1\end{array}$ & $\begin{array}{c}\mathrm{Cr} \\
\mathrm{ICP}-16\end{array}$ & $\begin{array}{c}\mathrm{Cu} \\
\mathrm{ICP}-40\end{array}$ & $\begin{array}{c}\text { Eu } \\
\text { ICP-40 }\end{array}$ & $\begin{array}{c}\mathrm{Ga} \\
\mathrm{ICP}-40\end{array}$ & $\begin{array}{c}\mathrm{Hg} \\
\text { CVAA }\end{array}$ & $\begin{array}{c}\mathrm{Ho} \\
\text { ICP-40 }\end{array}$ & $\begin{array}{c}\mathrm{La} \\
\mathrm{ICP}-40\end{array}$ & $\begin{array}{c}\mathrm{Li} \\
\mathrm{ICP}-40\end{array}$ & $\begin{array}{c}\mathrm{Mn} \\
\text { ICP-40 }\end{array}$ & $\begin{array}{c}\text { Mo } \\
\text { ICP-40 }\end{array}$ & $\begin{array}{c}\mathrm{Nb} \\
\text { ICP-40 }\end{array}$ & $\begin{array}{c}\mathrm{Nd} \\
\mathrm{ICP}-40\end{array}$ & $\begin{array}{c}\mathrm{Ni} \\
\text { ICP-40 }\end{array}$ & $\begin{array}{c}\mathrm{Pb} \\
\mathrm{ICP}-40\end{array}$ & $\begin{array}{c}\mathrm{Sb} \\
\text { Hydride }\end{array}$ \\
\hline$\overline{601-27-1 N}$ & 172 & 32 & $<2$ & $<4$ & 0.05 & $<4$ & 20 & 14 & 81 & $<2$ & $<4$ & 25 & 37 & 9 & 0.7 \\
\hline $601-27-1 \mathrm{M}$ & 759 & 56 & $<2$ & 14 & 0.15 & $<4$ & 31 & 27 & 76 & $<2$ & $<4$ & 37 & 97 & 7 & 0.6 \\
\hline $601-27-1 \mathrm{~L} 2$ & 95 & 29 & $<2$ & $<4$ & 0.02 & $<4$ & 8 & 16 & 298 & $<2$ & 6 & 10 & 14 & 4 & $<0.6$ \\
\hline $601-27-1 \mathrm{~L} 1$ & 137 & 20 & $<2$ & $<4$ & 0.04 & $<4$ & 10 & 29 & 333 & $<2$ & $<4$ & 13 & 45 & $<4$ & $<0.6$ \\
\hline $601-27-1 \mathrm{~K} 2$ & 139 & 23 & $<2$ & $<4$ & 0.04 & $<4$ & 10 & 17 & 68 & 3 & $<4$ & 14 & 29 & 5 & $<0.6$ \\
\hline $601-27-1 \mathrm{~K}$ & 224 & 49 & $<2$ & 4 & 0.03 & $<4$ & 16 & 13 & 60 & $<2$ & $<4$ & 22 & 40 & 7 & 0.6 \\
\hline $601-27-1 \mathrm{~J}$ & 300 & 31 & $<2$ & 5 & 0.03 & $<4$ & 17 & 12 & 91 & $<2$ & $<4$ & 22 & 44 & 6 & 0.9 \\
\hline 601-27-1I & 1100 & 36 & 8 & 8 & 0.14 & 7 & 176 & 43 & 31 & $<2$ & 4 & 197 & 21 & 13 & 2.2 \\
\hline $601-27-1 \mathrm{H}$ & 106 & 13 & $<2$ & $<4$ & 0.02 & $<4$ & 18 & 8 & 13 & $<2$ & $<4$ & 21 & 10 & $<4$ & $<0.6$ \\
\hline $601-27-1 \mathrm{G}$ & 170 & 23 & $<2$ & $<4$ & 0.04 & $<4$ & 14 & 8 & 16 & $<2$ & $<4$ & 30 & 13 & $<4$ & $<0.6$ \\
\hline $601-27-1 F$ & 23 & 11 & $<2$ & $<4$ & $<0.02$ & $<4$ & 12 & 5 & 16 & $<2$ & $<4$ & 12 & 4 & $<4$ & $<0.6$ \\
\hline $601-27-1 E$ & 67 & 27 & $<2$ & $<4$ & 0.05 & $<4$ & 19 & 6 & 38 & $<2$ & $<4$ & 23 & 21 & $<4$ & $<0.6$ \\
\hline 601-27-1D & 86 & 152 & $<2$ & $<4$ & 0.04 & $<4$ & 3 & 9 & 42 & 2 & $<4$ & $<9$ & 20 & 9 & 0.6 \\
\hline $601-27-1 C$ & 19 & 33 & $<2$ & $<4$ & $<0.02$ & $<4$ & 3 & 4 & 37 & $<2$ & $<4$ & $<9$ & 8 & $<4$ & $<0.6$ \\
\hline $601-27-1 B$ & 39 & 30 & $<2$ & $<4$ & $<0.02$ & $<4$ & 7 & 4 & 50 & $<2$ & $<4$ & $<9$ & 11 & $<4$ & $<0.6$ \\
\hline $601-27-1 \mathrm{~A}$ & 12 & 24 & $<2$ & $<4$ & $<0.02$ & $<4$ & 3 & 4 & 148 & $<2$ & $<4$ & $<9$ & 4 & 4 & $<0.6$ \\
\hline$\underline{\text { Section } 5}$ & & & & & & & & & & & & & & & \\
\hline$\overline{601-26-1} \mathrm{~A}$ & 51 & 24 & $<2$ & $<4$ & 0.02 & $<4$ & 5 & 6 & 29 & $<2$ & $<4$ & $<9$ & 8 & $<4$ & 0.9 \\
\hline 601-26-1B & 72 & 60 & $<2$ & $<4$ & 0.1 & $<4$ & 10 & 10 & 1710 & $<2$ & $<4$ & 15 & 98 & 5 & 1 \\
\hline $601-26-1 C$ & 81 & 32 & $<2$ & $<4$ & 0.03 & $<4$ & 4 & 13 & 36 & $<2$ & $<4$ & 11 & 11 & $<4$ & 0.7 \\
\hline
\end{tabular}


Table 3 continued

\begin{tabular}{|c|c|c|c|c|c|c|c|c|c|c|c|c|c|c|c|}
\hline $\begin{array}{l}\text { Sample No. } \\
\text { Section } 5\end{array}$ & $\begin{array}{c}\mathrm{Cr} \\
\mathrm{ICP}-16\end{array}$ & $\begin{array}{c}\mathrm{Cu} \\
\mathrm{ICP}-40\end{array}$ & $\begin{array}{c}\mathrm{Eu} \\
\mathrm{ICP}-40\end{array}$ & $\begin{array}{c}\mathrm{Ga} \\
\mathrm{ICP}-40\end{array}$ & $\begin{array}{c}\mathrm{Hg} \\
\text { CVAA }\end{array}$ & $\begin{array}{c}\text { Ho } \\
\text { ICP-40 }\end{array}$ & $\begin{array}{c}\mathrm{La} \\
\mathrm{ICP}-40\end{array}$ & $\begin{array}{c}\mathrm{Li} \\
\mathrm{ICP}-40\end{array}$ & $\begin{array}{c}\mathrm{Mn} \\
\mathrm{ICP}-40\end{array}$ & $\begin{array}{c}\text { Mo } \\
\text { ICP-40 }\end{array}$ & $\begin{array}{c}\mathrm{Nb} \\
\mathrm{ICP}-40\end{array}$ & $\begin{array}{c}\mathrm{Nd} \\
\mathrm{ICP}-40\end{array}$ & $\begin{array}{c}\mathrm{Ni} \\
\text { ICP-40 }\end{array}$ & $\begin{array}{c}\mathrm{Pb} \\
\mathrm{ICP}-40\end{array}$ & $\begin{array}{c}\text { Sb } \\
\text { Hydride }\end{array}$ \\
\hline $\begin{array}{l}601-26-1 \mathrm{D} \\
\text { Section } 7\end{array}$ & 74 & 13 & $<2$ & $<4$ & 0.03 & $<4$ & 6 & 10 & 22 & $<2$ & $<4$ & 9 & 13 & $<4$ & 0.7 \\
\hline 601-28-1R1 & 15 & 9 & $<2$ & $<4$ & $<0.02$ & $<4$ & 3 & 5 & 106 & $<2$ & $<4$ & $<9$ & 6 & $<4$ & 0.8 \\
\hline 601-28-1Q & 12 & 6 & $<2$ & $<4$ & $<0.02$ & $<4$ & 3 & 4 & 21 & $<2$ & $<4$ & $<9$ & 5 & $<4$ & $<0.6$ \\
\hline $502-14-2 \mathrm{G}$ & 16 & 5 & $<2$ & $<4$ & $<0.02$ & $<4$ & 7 & 4 & 48 & $<2$ & $<4$ & $<9$ & 6 & $<4$ & $<0.6$ \\
\hline $502-14-2 \mathrm{~F}$ & 36 & 8 & $<2$ & $<4$ & 0.03 & $<4$ & 9 & 4 & 23 & $<2$ & $<4$ & $<9$ & 7 & $<4$ & $<0.6$ \\
\hline $502-14-2 \mathrm{E}$ & 50 & 5 & $<2$ & $<4$ & 0.02 & $<4$ & 9 & 6 & 143 & $<2$ & $<4$ & $<9$ & 16 & $<4$ & $<0.6$ \\
\hline $601-28-1 \mathrm{P}$ & $<10$ & 10 & $<2$ & $<4$ & $<0.02$ & $<4$ & 3 & 4 & 160 & $<2$ & $<4$ & $<9$ & 23 & $<4$ & $<0.6$ \\
\hline $601-28-1 \mathrm{~N}$ & 20 & 148 & $<2$ & $<4$ & $<0.02$ & $<4$ & 5 & 5 & 92 & $<2$ & $<4$ & $<9$ & 8 & $<4$ & $<0.6$ \\
\hline $601-28-1 \mathrm{M}$ & $<10$ & 8 & $<2$ & $<4$ & $<0.02$ & $<4$ & 3 & $<2$ & 57 & $<2$ & $<4$ & $<9$ & 4 & $<4$ & $<0.6$ \\
\hline $601-28-1 \mathrm{~L}$ & 10 & 93 & $<2$ & $<4$ & $<0.02$ & $<4$ & 4 & 3 & 191 & $<2$ & $<4$ & $<9$ & 10 & $<4$ & $<0.6$ \\
\hline $601-28-1 \mathrm{~K}$ & 15 & 40 & $<2$ & $<4$ & $<0.02$ & $<4$ & 6 & 13 & 221 & $<2$ & $<4$ & $<9$ & 11 & $<4$ & $<0.6$ \\
\hline $601-28-1 \mathrm{~J} 1$ & 12 & 122 & $<2$ & $<4$ & $<0.02$ & $<4$ & 4 & 4 & 299 & $<2$ & $<4$ & $<9$ & 20 & $<4$ & 1 \\
\hline $601-28-1 J 2$ & 11 & 7 & $<2$ & $<4$ & $<0.02$ & $<4$ & 3 & 4 & 250 & $<2$ & $<4$ & $<9$ & 15 & $<4$ & $<0.6$ \\
\hline $601-28-1 \mathrm{~J} 3$ & 13 & 12 & $<2$ & $<4$ & $<0.02$ & $<4$ & 6 & 7 & 227 & $<2$ & $<4$ & $<9$ & 21 & $<4$ & $<0.6$ \\
\hline 601-28-1I & 48 & 10 & $<2$ & $<4$ & 0.03 & $<4$ & 9 & 6 & 48 & $<2$ & $<4$ & $<9$ & 13 & $<4$ & 0.7 \\
\hline $601-28-1 \mathrm{H}$ & 13 & 33 & $<2$ & $<4$ & 0.03 & $<4$ & 6 & 6 & 107 & $<2$ & $<4$ & $<9$ & 13 & 58 & 11.4 \\
\hline 601-28-1G1 & 148 & 154 & $<2$ & $<4$ & 0.04 & $<4$ & 18 & 12 & 55 & $<2$ & $<4$ & 13 & 31 & 6 & 1 \\
\hline $601-28-1 \mathrm{G} 2$ & 102 & 34 & $<2$ & $<4$ & 0.06 & $<4$ & 12 & 8 & 51 & 2 & $<4$ & $<9$ & 29 & $<4$ & $<0.6$ \\
\hline $601-28-1 \mathrm{G} 3$ & 234 & 296 & $<2$ & $<4$ & 0.05 & $<4$ & 29 & 16 & 58 & $<2$ & $<4$ & 21 & 45 & $<4$ & 0.8 \\
\hline $601-28-1 F$ & 601 & 52 & $<2$ & 6 & 0.05 & $<4$ & 48 & 18 & 34 & $<2$ & $<4$ & 37 & 86 & 5 & 1.5 \\
\hline $601-28-1 E$ & 452 & 38 & $<2$ & 16 & 0.16 & $<4$ & 29 & 21 & 32 & 12 & $<4$ & 18 & 33 & 14 & 1.8 \\
\hline 601-28-1D & 135 & 52 & $<2$ & 13 & 0.1 & $<4$ & 26 & 18 & 24 & $<2$ & 5 & 20 & 25 & 13 & 0.9 \\
\hline $601-28-1 C$ & 589 & 67 & 4 & 12 & 0.17 & $<4$ & 140 & 21 & 496 & 14 & 5 & 93 & 251 & 13 & 1.8 \\
\hline $502-14-2 \mathrm{D}$ & 151 & 36 & $<2$ & $<4$ & 0.08 & $<4$ & 92 & 3 & 10 & 6 & $<4$ & 57 & 40 & 13 & $<0.6$ \\
\hline $502-14-2 \mathrm{C} 1$ & 73 & 17 & $<2$ & $<4$ & 0.03 & $<4$ & 8 & $<2$ & 102 & 7 & $<4$ & 10 & 16 & 6 & $<0.6$ \\
\hline $502-14-2 B$ & 1180 & 98 & 3 & $<4$ & 0.3 & $<4$ & 141 & 7 & 59 & 24 & 4 & 81 & 223 & 12 & 4.1 \\
\hline $601-28-1 B$ & 153 & 41 & $<2$ & $<4$ & 0.03 & $<4$ & 11 & $<2$ & 69 & 18 & 4 & 16 & 27 & 6 & 0.9 \\
\hline $502-14-2 \mathrm{~A}$ & 1650 & 184 & $<2$ & 8 & 0.64 & $<4$ & 86 & 14 & 82 & 117 & 6 & 53 & 387 & 15 & 9.2 \\
\hline $601-28-1 \mathrm{~A}$ & 1800 & 183 & 2 & 15 & 0.59 & $<4$ & 84 & 31 & 146 & 65 & 11 & 59 & 361 & 14 & 6.2 \\
\hline $\begin{array}{l}\text { Sample No. } \\
\text { Section } 1\end{array}$ & $\begin{array}{c}\mathrm{Sc} \\
\mathrm{ICP}-40\end{array}$ & $\begin{array}{c}\mathrm{Se} \\
\text { Hydride }\end{array}$ & $\begin{array}{c}\mathrm{Sr} \\
\text { ICP-40 }\end{array}$ & $\begin{array}{c}\mathrm{Sr} \\
\text { ICP-16 }\end{array}$ & $\begin{array}{c}\text { Th } \\
\text { ICP-40 }\end{array}$ & $\begin{array}{c}\mathrm{Tl} \\
\text { Hydride }\end{array}$ & $\begin{array}{c}\mathrm{V} \\
\text { ICP-40 }\end{array}$ & $\begin{array}{c}\mathrm{Y} \\
\mathrm{ICP}-40\end{array}$ & $\begin{array}{c}\mathrm{Yb} \\
\mathrm{ICP}-40\end{array}$ & $\begin{array}{c}\mathrm{Zn} \\
\text { ICP-40 }\end{array}$ & $\begin{array}{c}\mathrm{Zr} \\
\mathrm{ICP}-16\end{array}$ & & & & \\
\hline $601-27-1 \mathrm{~N}$ & 3 & 2.8 & 85 & 92 & $<6$ & 0.1 & 32 & 36 & 2 & 110 & 95 & & & & \\
\hline $601-27-1 \mathrm{M}$ & 9 & 0.9 & 66 & 70 & 7 & 0.5 & 86 & 49 & 3 & 154 & 164 & & & & \\
\hline 601-27-1L2 & $<2$ & 0.4 & 124 & 126 & $<6$ & $<0.1$ & 14 & 13 & $<1$ & 28 & 31 & & & & \\
\hline 601-27-1L1 & 3 & 0.9 & 23 & 26 & $<6$ & 0.3 & 27 & 20 & 2 & 112 & 70 & & & & \\
\hline $601-27-1 \mathrm{~K} 2$ & 2 & 1.8 & 64 & 65 & $<6$ & 0.1 & 20 & 16 & $<1$ & 49 & 72 & & & & \\
\hline $601-27-1 \mathrm{~K}$ & 4 & 1.9 & 52 & 56 & $<6$ & 0.1 & 31 & 31 & 2 & 98 & 101 & & & & \\
\hline $601-27-1 \mathrm{~J}$ & 4 & 2.4 & 63 & 65 & $<6$ & 0.1 & 37 & 32 & 2 & 99 & 91 & & & & \\
\hline 601-27-1I & 3 & 2.2 & 215 & 231 & $<6$ & 0.1 & 188 & 321 & 13 & 52 & 42 & & & & \\
\hline $601-27-1 \mathrm{H}$ & $<2$ & 0.9 & 27 & 30 & $<6$ & $<0.1$ & 24 & 30 & 1 & 43 & $<10$ & & & & \\
\hline $601-27-1 \mathrm{G}$ & $<2$ & 1.2 & 34 & 40 & $<6$ & 0.3 & 38 & 54 & 3 & 135 & 18 & & & & \\
\hline
\end{tabular}


Table 3 continued

\begin{tabular}{|c|c|c|c|c|c|c|c|c|c|c|c|}
\hline $\begin{array}{l}\text { Sample No. } \\
\text { Section } 1 \\
\end{array}$ & $\begin{array}{c}\mathrm{Sc} \\
\mathrm{ICP}-40\end{array}$ & $\begin{array}{c}\text { Se } \\
\text { Hydride }\end{array}$ & $\begin{array}{c}\mathrm{Sr} \\
\mathrm{ICP}-40\end{array}$ & $\begin{array}{c}\mathrm{Sr} \\
\mathrm{ICP}-16\end{array}$ & $\begin{array}{c}\text { Th } \\
\text { ICP-40 }\end{array}$ & $\begin{array}{c}\mathrm{Tl} \\
\text { Hydride }\end{array}$ & $\begin{array}{c}\mathrm{V} \\
\mathrm{ICP}-40\end{array}$ & $\begin{array}{c}\mathrm{Y} \\
\mathrm{ICP}-40\end{array}$ & $\begin{array}{c}\mathrm{Yb} \\
\mathrm{ICP}-40\end{array}$ & $\begin{array}{c}\mathrm{Zn} \\
\mathrm{ICP}-40\end{array}$ & $\begin{array}{c}\mathrm{Zr} \\
\mathrm{ICP}-16\end{array}$ \\
\hline $601-27-1 \mathrm{~F}$ & $<2$ & 0.4 & 16 & 17 & $<6$ & $<0.1$ & 4 & 16 & $<1$ & 30 & $<10$ \\
\hline $601-27-1 E$ & $<2$ & 1.9 & 44 & 47 & $<6$ & $<0.1$ & 21 & 46 & 2 & 78 & 15 \\
\hline 601-27-1D & $<2$ & 0.8 & 14 & 14 & $<6$ & $<0.1$ & 19 & 8 & $<1$ & 133 & $<10$ \\
\hline $601-27-1 \mathrm{C}$ & $<2$ & $<0.2$ & 23 & 23 & $<6$ & $<0.1$ & 5 & 6 & $<1$ & 57 & 13 \\
\hline $601-27-1 B$ & $<2$ & 0.3 & 34 & 36 & $<6$ & $<0.1$ & 14 & 13 & $<1$ & 81 & 16 \\
\hline $601-27-1 \mathrm{~A}$ & $<2$ & $<0.2$ & 188 & 183 & $<6$ & $<0.1$ & 5 & 3 & $<1$ & 32 & $<10$ \\
\hline \multicolumn{12}{|l|}{$\underline{\text { Section } 5}$} \\
\hline$\overline{601-26-1} \mathrm{~A}$ & $<2$ & 0.6 & 22 & 23 & $<6$ & $<0.1$ & 11 & 10 & $<1$ & 29 & $<10$ \\
\hline 601-26-1B & $<2$ & 0.6 & 24 & 28 & $<6$ & 0.3 & 35 & 20 & 1 & 244 & 45 \\
\hline $601-26-1 \mathrm{C}$ & $<2$ & 0.5 & 24 & 27 & $<6$ & $<0.1$ & 15 & 12 & $<1$ & 45 & 27 \\
\hline 601-26-1D & $<2$ & 0.9 & 51 & 54 & $<6$ & $<0.1$ & 18 & 13 & $<1$ & 50 & 23 \\
\hline \multicolumn{12}{|l|}{ Section 7} \\
\hline$\overline{601-28-1 R}$ R & $<2$ & $<0.2$ & 19 & 21 & $<6$ & $<0.1$ & 5 & 5 & $<1$ & 38 & 12 \\
\hline $601-28-1 Q$ & $<2$ & 0.2 & 17 & 17 & $<6$ & $<0.1$ & 3 & 5 & $<1$ & 24 & $<10$ \\
\hline $502-14-2 \mathrm{G}$ & $<2$ & 0.4 & 22 & 22 & $<6$ & $<0.1$ & 5 & 10 & $<1$ & 35 & 13 \\
\hline $502-14-2 \mathrm{~F}$ & $<2$ & $<0.2$ & 21 & 22 & $<6$ & $<0.1$ & 9 & 13 & $<1$ & 36 & 22 \\
\hline $502-14-2 \mathrm{E}$ & $<2$ & 0.8 & 23 & 24 & $<6$ & $<0.1$ & 14 & 13 & $<1$ & 63 & 21 \\
\hline $601-28-1 P$ & $<2$ & $<0.2$ & 15 & 15 & $<6$ & $<0.1$ & $<2$ & 4 & $<1$ & 73 & $<10$ \\
\hline $601-28-1 \mathrm{~N}$ & $<2$ & $<0.2$ & 11 & 15 & $<6$ & $<0.1$ & 3 & 7 & $<1$ & 121 & 13 \\
\hline $601-28-1 \mathrm{M}$ & $<2$ & $<0.2$ & 8 & $<10$ & $<6$ & $<0.1$ & $<2$ & 3 & $<1$ & 28 & $<10$ \\
\hline $601-28-1 \mathrm{~L}$ & $<2$ & $<0.2$ & 10 & 11 & $<6$ & $<0.1$ & 3 & 6 & $<1$ & 109 & 12 \\
\hline $601-28-1 \mathrm{~K}$ & $<2$ & $<0.2$ & 12 & 13 & $<6$ & $<0.1$ & 5 & 8 & $<1$ & 94 & 21 \\
\hline 601-28-1J1 & $<2$ & $<0.2$ & 14 & 15 & $<6$ & $<0.1$ & 3 & 6 & $<1$ & 153 & $<10$ \\
\hline $601-28-1 \mathrm{~J} 2$ & $<2$ & $<0.2$ & 12 & 15 & $<6$ & $<0.1$ & $<2$ & 4 & $<1$ & 62 & $<10$ \\
\hline $601-28-1 \mathrm{~J} 3$ & $<2$ & $<0.2$ & 15 & 17 & $<6$ & $<0.1$ & 4 & 8 & $<1$ & 79 & $<10$ \\
\hline 601-28-1I & $<2$ & 2.5 & 29 & 32 & $<6$ & $<0.1$ & 13 & 15 & $<1$ & 91 & 14 \\
\hline $601-28-1 \mathrm{H}$ & $<2$ & 0.7 & 16 & 17 & $<6$ & $<0.1$ & 5 & 9 & $<1$ & 90 & $<10$ \\
\hline 601-28-1G1 & $<2$ & 1.3 & 43 & 46 & $<6$ & 0.7 & 47 & 29 & 1 & 253 & 19 \\
\hline $601-28-1 \mathrm{G} 2$ & $<2$ & 2.9 & 36 & 35 & $<6$ & 0.4 & 27 & 19 & $<1$ & 123 & 13 \\
\hline $601-28-1 \mathrm{G} 3$ & 3 & 1.3 & 58 & 60 & $<6$ & 0.8 & 63 & 44 & 2 & 437 & 24 \\
\hline $601-28-1 F$ & 4 & 3.8 & 95 & 97 & $<6$ & 0.9 & 130 & 76 & 4 & 569 & 52 \\
\hline $601-28-1 \mathrm{E}$ & 8 & 138 & 80 & 83 & 12 & 1.3 & 122 & 10 & 2 & 96 & 394 \\
\hline 601-28-1D & 7 & 101 & 78 & 82 & 9 & 1 & 63 & 9 & 2 & 115 & 397 \\
\hline $601-28-1 \mathrm{C}$ & 9 & 17.2 & 275 & 282 & $<6$ & 1.1 & 110 & 206 & 10 & 658 & 233 \\
\hline $502-14-2 \mathrm{D}$ & 4 & 114 & 920 & 914 & $<6$ & 2.6 & 579 & 191 & 10 & 483 & 62 \\
\hline $502-14-2 \mathrm{C} 1$ & $<2$ & 16.8 & 425 & 420 & $<6$ & 0.8 & 176 & 13 & $<1$ & 284 & 30 \\
\hline $502-14-2 B$ & 6 & 57.1 & 1010 & 1030 & $<6$ & 1 & 486 & 256 & 11 & 1070 & 114 \\
\hline $601-28-1 B$ & $<2$ & 6.1 & 696 & 699 & $<6$ & $<0.1$ & 280 & 21 & $<1$ & 201 & 23 \\
\hline $502-14-2 \mathrm{~A}$ & 6 & 83.9 & 749 & 788 & $<6$ & 2.4 & 586 & 147 & 8 & 3060 & 134 \\
\hline $601-28-1 \mathrm{~A}$ & 9 & 99.5 & 437 & 436 & $<6$ & 1.1 & 196 & 144 & 8 & 1460 & 243 \\
\hline
\end{tabular}


measured as evolved $\mathrm{CO}_{2}$ after acidification of the sample, and organic carbon was calculated as the difference between total and carbonate carbon. The compilations by Arbogast (1996) and Baedecker (1987) include additional discussions about the various types of analytical methodologies used here.

The concentration of each element is reported in the chemistry table as received from the analysts. However, qualified data (detection limit values) were modified for use in

statistical analyses. An element was not used in statistical analyses if more than $30 \%$ of the data points for that particular element were qualified. If there were fewer than $30 \%$ qualified values for an element, then the qualified values were multiplied by 0.5 and data for that element were used in the statistical analyses.

Mineral compositions were determined by X-ray diffraction using a Philips diffractometer with a graphite monochromator and $\mathrm{CuK} \alpha$ radiation. Samples were run from $4-70^{\circ} 2 \Theta$ at $40 \mathrm{kV}, 45 \mathrm{~mA}$, and 10 counts per second. Semiquantitative mineral contents were determined and are grouped in Table 2 under the classifications of major $(>25 \%)$, moderate $(5-25 \%)$, and minor $(<5 \%)$.

Statistical analyses were performed on 3 data sets including all data (except data for the Meade Peak in section 7 and the two carbonate beds in section 1); data solely for section 1 and data solely for section 7 . The usual Pearson product moment correlation coefficient was used to calculate correlation coefficient matrices. A 99\% confidence level was used to calculate the zero-point of correlation. For Q-mode factor analysis, each variable percentage was scaled to the percent of the maximum value before the values were rownormalized and cosine theta coefficients calculated. Factors were derived from orthogonal rotations of principal component eigenvectors using the Varimax method (Klovan and Imbrie, 1971). All communalities are $\geq 0.90$.

\section{RESULTS}

\section{Petrography}

The dominant characteristic of the chert beds is the presence of sponge spicules, which vary from relatively well preserved to faint ghosts. Most of the chert beds can be classified as spicularites (spongolites). These spicularites are laminated and commonly show a preferred orientation of elongate grains parallel to bedding. One sample (601-28-1H) shows preferred orientation in some laminae but not in others, indicating that the alignment of grains was caused by bottom currents rather than by compaction or tectonics. The sparse to common occurrence of rhombs characterize most spicularite beds. These rhombs are likely quartz-replaced dolomite rhombs. Glauconite, mica, and feldspar are present in some beds. Various combinations of bivalves, fish debris, radiolarians(?), and calcareous algae(?), are seen in some beds. Spicularite beds in section 7 show sedimentary structures that include cross bedding and cut-and-fill scouring.

The upper part of section 7 does not consist of spicularite beds, but rather consist of replaced carbonates. These chert beds are generally white to grayish, centimeters to a meter thick, and in thin section consist of abundant rhombs partly to completely replaced by quartz. Some replaced rhombs show relict carbonate twinning. Laminae are compacted around some large rhombs, which indicates that they formed during early diagenesis prior to compaction. The textures indicate that carbonate and silica fossils were deposited on the seafloor, dolomite rhombs formed in unconsolidated sediment during early diagenesis, compaction took place with increasing burial, and finally carbonate grains were replaced and cement was precipitated during silica diagenesis, thereby producing chert. 
Table 2. X-Ray diffraction mineralogy of Rex Chert, cherty shale, and Meade Peak samples

\begin{tabular}{|c|c|c|c|c|}
\hline SAMPLE & LITHOLOGY & MAJOR & MODERATE & MINOR/ TRACE \\
\hline $\begin{array}{l}\text { Section } 1 \\
601-27-1 \mathrm{~N}\end{array}$ & Cherty-calcareous shale bed & quartz & feldspar & dolomite, gypsum, smectite(?) \\
\hline $601-27-1 \mathrm{M}$ & Laminated siliceous shale & quartz & feldspar & illite, smectite, CFA \\
\hline 601-27-1L2 & Siliceous dolostone & quartz & dolomite & calcite, feldspar, smectite(?) \\
\hline 601-27-1L1 & Argillaceous chert bed & quartz & feldspar & goethite \\
\hline $601-27-1 \mathrm{~K} 2$ & Argillaceous chert bed & quartz & -- & dolomite, feldspar, smectite, gypsum(?) \\
\hline $601-27-1 \mathrm{~K}$ & Shaly interbed & quartz & -- & feldspar, smectite, illite, CFA \\
\hline $601-27-1 \mathrm{~J}$ & Cherty shale bed & quartz & -- & feldspar, illite, CFA, calcite, dolomite \\
\hline $601-27-1 I$ & Phosphatic-ferruginous chert bed & quartz & CFA & clay mineral \\
\hline $601-27-1 \mathrm{H}$ & Chert bed & quartz & -- & illite, CFA, smectite(?) \\
\hline $601-27-1 G$ & Base of massive thick chert bed & quartz & -- & CFA, heulandite(?) \\
\hline $601-27-1 \mathrm{~F}$ & Chert bed & quartz & -- & smectite(?) \\
\hline $601-27-1 \mathrm{E}$ & Chert bed & quartz & -- & CFA, smectite(?), chlorite or kaolinite(?) \\
\hline $601-27-1 \mathrm{D}$ & Chert bed & quartz & -- & clay mineral \\
\hline $601-27-1 \mathrm{C}$ & Chert bed & quartz & -- & dolomite, calcite, smectite(?) \\
\hline $601-27-1 B$ & Chert bed & quartz & -- & dolomite, calcite, smectite(?) \\
\hline $601-27-1 \mathrm{~A}$ & Siliceous limestone bed & calcite & quartz & dolomite, smectite(?) \\
\hline \multicolumn{5}{|l|}{ Section 5} \\
\hline $601-26-1 \mathrm{~A}$ & Chert bed & quartz & -- & -- \\
\hline $601-26-1 B$ & Leached zone in chert & quartz & -- & kaolinite or chlorite, goethite, bixbyite \\
\hline $601-26-1 C$ & Chert bed & quartz & -- & illite, chlorite or kaolinite \\
\hline $601-26-1 \mathrm{D}$ & Chert bed & quartz & -- & illite, chlorite or kaolinite \\
\hline \multicolumn{5}{|l|}{ Section 7} \\
\hline 601-28-1R1 & Breccia & quartz & -- & dolomite \\
\hline 601-28-1Q & Chert bed & quartz & -- & CFA(?) \\
\hline $502-14-2 \mathrm{G}$ & Chert bed & quartz & -- & CFA \\
\hline $502-14-2 \mathrm{~F}$ & Chert bed & quartz & -- & CFA, clay minerals \\
\hline $502-14-2 \mathrm{E}$ & Chert bed & uartz & -- & CFA, clay minerals \\
\hline $601-28-1 P$ & Chert bed & quartz & -- & calcite, dolomite, clay minerals \\
\hline $601-28-1 \mathrm{~N}$ & $1 \mathrm{~N}-1 \mathrm{~K}=$ parts of a $80 \mathrm{~cm}$ thick & quartz & -- & -- \\
\hline $601-28-1 \mathrm{M}$ & white chert bed & quartz & -- & clay minerals \\
\hline $601-28-1 \mathrm{~L}$ & chert & quartz & -- & smectite(?) \\
\hline $601-28-1 \mathrm{~K}$ & chert & quartz & -- & smectite(?) \\
\hline $601-28-1 \mathrm{~J} 3$ & Chert & quartz & -- & CFA(?) \\
\hline $601-28-1 \mathrm{~J} 2$ & Chert & quartz & -- & -- \\
\hline $601-28-1 \mathrm{~J} 1$ & Chert & quartz & -- & smectite(?) \\
\hline 601-28-1I & Chert bed & quartz & -- & $\mathrm{CFA}(?)$ \\
\hline $601-28-1 \mathrm{H}$ & Chert bed & quartz & -- & CFA(?), smectite(?) \\
\hline 601-28-1G1 & Chert & quartz & -- & CFA(?), gypsum \\
\hline 601-28-1G2 & Chert & quartz & -- & CFA, clay minerals \\
\hline 601-28-1G3 & Chert & quartz & -- & CFA, smectite \\
\hline $601-28-1 \mathrm{~F}$ & Phosphatic cherty shale bed & quartz & CFA & illite, smectite(?) \\
\hline $601-28-1 \mathrm{E}$ & Siliceous siltstone & quartz & plagioclase & Illite \\
\hline $601-28-1 \mathrm{D}$ & Siliceous siltstone, marker bed & quartz & feldspar & illite, smectite(?), jarosite(?) \\
\hline $601-28-1 \mathrm{C}$ & Phosphatic-calcareous shale & quartz & CFA, calcite, feldspar & illite, natrojarosite \\
\hline $502-14-2 \mathrm{D}$ & Phosphorite & CFA & -- & quartz, clay minerals \\
\hline $502-14-2 \mathrm{C}$ & Carbonate nodule in black shale & calcite & CFA, dolomite & -- \\
\hline $502-14-2 B$ & Phosphatic shale & CFA & calcite, quartz & dolomite, plagioclase, illite \\
\hline $601-28-1 B$ & Carbonate nodule in black shale & calcite & dolomite, CFA & quartz \\
\hline $502-14-2 \mathrm{~A}$ & Phosphatic shale & CFA, calcite & quartz, plagioclase & pyrite (?), dolomite, mica, clay minerals \\
\hline $601-28-1 \mathrm{~A}$ & Black phosphatic shale & quartz & feldspar, CFA & calcite, dolomite, illite, pyrite \\
\hline
\end{tabular}


The cherty shale member from section 1 consists of siliceous siltstone beds and one siliceous dolostone bed. Beds are laminated, contain ghosts of spicules, and some beds contain various combinations of sparse rhombs, bivalves, fish debris, feldspar, fibrous clay minerals, and calcite. Carbonate minerals are more common than they are in the underlying chert. Grains are well sorted. Uncommon sedimentary structures include burrows and reverse grading.

The siliceous siltstone from the Meade Peak-Rex Chert transition zone in section 7 is somewhat different from siliceous siltstone in section 1 . The section 7 siltstone is more compacted and shows a preferred fabric created by aggregate extinction of clay minerals, or by parallel orientation of thin wavy iron-rich or organic-matter-rich lenses. Grains are moderately well sorted and range from angular to subrounded, but are predominantly subangular. Similar to section 1, siltstone beds are laminated, contain ghosts of spicules, and some beds contain various combinations of bivalves, fish debris, feldspar, and fibrous clay minerals, as well as mica and chlorite; however no rhombs or calcite were seen.

\section{Mineralogy}

The mineral content of the Rex Chert and cherty shale member is dominated by quartz (Table 2). Several beds also have major amounts of carbonate minerals: the lowermost bed in section 1 (601-27-1A), which contains major calcite as well as quartz; and a siliceous dolostone (601-27-1L2) in section 1 contains major dolomite as well as quartz. Feldspar (combined K-feldspar and plagioclase), dolomite, and carbonate fluorapatite (CFA) occur in moderate amounts in a few beds (Table 2). Clay minerals occur in minor amounts. In the upper part of the Meade Peak Member in section 7, calcite and CFA are major phases along with quartz in some samples.

\section{Chemical Composition}

The mean concentrations of elements in the Rex Chert and the cherty shale member are overwhelmingly dominated by silica, which averages $94.6 \%$ for the three sections studied and ranges from $92.2 \%$ for section 1 to $96.9 \%$ for section 5 (Table 3 ). The sums of the major oxides are reasonable close to $100 \%$, but are a little high for most of the stratigraphically highest 13 samples from section 7, up to $103 \%$ (Table 3). These high values provide a measure of the analytical accuracy of silica determinations for these very high silica rocks. Samples with low sums of the major oxides results from not including organic carbon and sulfur compounds in those totals, which occur predominantly for the Meade Peak rocks in section 7.

Organic carbon contents are generally very low in the chert, but are up to $1.8 \%$ in samples from the cherty shale member in section 1 and up to $3.4 \%$ in rocks from the transition between the Meade Peak and Rex Chert in section 7 (Table 3). Likewise, phosphate $\left(\mathrm{P}_{2} \mathrm{O}_{5}\right)$ is generally very low in the chert, but can be high (up to $3.1 \%$ ) in individual beds; these beds do not consistently occur at any particular stratigraphic level in the sections.

Selenium concentrations for the Rex Chert and cherty shale member vary from the detection limit $(<0.2)$ to $138 \mathrm{ppm}$, with a mean concentration of $7 \mathrm{ppm}$ (Table 4), or $<1.0$ ppm if two outliers are removed.

Mean Se concentrations vary from $0.65 \mathrm{ppm}$ in section 5 (Tables 6) to $12 \mathrm{ppm}$ for section 7 (Table 7; this does not include values from Meade Peak rocks). The mean Se concentration for section 5 rocks is equivalent to that of mean shale, 0.6 ppm (Krauskopf, 1979), whereas that of section 1 (Table 5) is 2.2 times the concentration in mean shale and section 7 is 20 times the mean shale concentration. The reason for these differences is that section 7 includes the lowermost Rex Chert, which contains rocks of transitional character, and section 1 includes the cherty shale member. All the chert beds in the upper part of the Rex Chert in section 7 have Se concentrations of $<0.2$ to $0.8 \mathrm{ppm}$ and the mean concentration of 
Table 4. Statistics for 39 Rex Chert and cherty shale samples collected from measured sections 1,5 , and 7

\begin{tabular}{|c|c|c|c|c|c|c|}
\hline & $\mathbf{N}$ & Mean & Median & $\mathbf{S D}^{1}$ & Minimum & Maximum \\
\hline $\mathrm{SiO}_{2} \quad \mathrm{wt} \%$ & 39 & 94.6 & 96.3 & 6.79 & 74.9 & 102 \\
\hline $\mathrm{Al}_{2} \mathrm{O}_{3}$ & 39 & 1.82 & 0.72 & 2.64 & 0.23 & 11.1 \\
\hline $\mathrm{Fe}_{2} \mathrm{O}_{3}$ & 39 & 0.70 & 0.31 & 0.96 & 0.04 & 4.68 \\
\hline $\mathrm{TiO}_{2}$ & 39 & 0.11 & 0.03 & 0.19 & 0.01 & 0.82 \\
\hline $\mathrm{CaO}$ & 39 & 0.88 & 0.46 & 0.86 & 0.17 & 3.69 \\
\hline $\mathrm{K}_{2} \mathrm{O}$ & 39 & 0.35 & 0.10 & 0.63 & 0.005 & 2.58 \\
\hline $\mathrm{MgO}$ & 39 & 0.25 & 0.08 & 0.32 & 0.01 & 1.09 \\
\hline $\mathrm{Na}_{2} \mathrm{O}$ & 39 & 0.19 & 0.05 & 0.31 & 0.01 & 1.32 \\
\hline $\mathrm{P}_{2} \mathrm{O}_{5}$ & 39 & 0.42 & 0.25 & 0.54 & 0.05 & 3.05 \\
\hline$\sum \mathrm{CO}_{2}$ & 39 & 0.31 & 0.02 & 0.67 & 0.005 & 2.78 \\
\hline Total & 39 & 99.7 & 99.9 & 2.51 & 93.1 & 103 \\
\hline $\mathrm{C}_{\mathrm{t}}$ & 39 & 0.42 & 0.17 & 0.65 & 0.01 & 3.35 \\
\hline $\mathrm{C}_{\mathrm{c}}$ & 39 & 0.09 & 0.01 & 0.18 & 0.0015 & 0.76 \\
\hline $\mathrm{C}_{\text {org }}$ & 39 & 0.33 & 0.12 & 0.61 & 0.007 & 3.347 \\
\hline $\mathrm{S}_{\mathrm{t}}$ & 39 & 0.05 & 0.025 & 0.07 & 0.025 & 0.42 \\
\hline ppm & 39 & 4.22 & 2.50 & 4.45 & 0.30 & 16.8 \\
\hline $\mathrm{Ba}$ & 39 & 112 & 50 & 143 & 17 & 593 \\
\hline $\mathrm{Ba}$ & 39 & 111 & 49 & 140 & 16 & 611 \\
\hline $\mathrm{Ce}$ & 39 & 11 & 3 & 17 & 2.5 & 87 \\
\hline $\mathrm{Cr}$ & 39 & 143 & 67 & 227 & 5 & 1100 \\
\hline $\mathrm{Cu}$ & 39 & 46 & 31 & 58 & 5 & 296 \\
\hline $\mathrm{Hg}$ & 39 & 0.04 & 0.03 & 0.04 & 0.01 & 0.16 \\
\hline $\mathrm{La}$ & 39 & 16 & 9 & 28 & 3 & 176 \\
\hline $\mathrm{Li}$ & 39 & 10 & 8 & 8 & 1 & 43 \\
\hline $\mathrm{Mn}$ & 39 & 128 & 55 & 272 & 13 & 1710 \\
\hline $\mathrm{Nd}$ & 39 & 17 & 5 & 31 & 4.5 & 197 \\
\hline $\mathrm{Ni}$ & 39 & 25 & 16 & 23 & 4 & 98 \\
\hline $\mathrm{Sb}$ & 39 & 0.9 & 0.3 & 1.8 & 0.3 & 11.4 \\
\hline $\mathrm{Se}$ & 39 & 7.1 & 0.8 & 26.8 & 0.1 & 138 \\
\hline $\mathrm{Sr}$ & 39 & 39 & 24 & 37 & 8 & 215 \\
\hline V & 39 & 30 & 15 & 40 & 1 & 188 \\
\hline Y & 39 & 26 & 13 & 51 & 2 & 321 \\
\hline $\mathrm{Zn}$ & 39 & 110 & 90 & 107 & 24 & 569 \\
\hline $\mathrm{Zr}$ & 39 & 48 & 16 & 89 & 5 & 397 \\
\hline
\end{tabular}

${ }^{1}$ Standard Deviation 
Table 5. Statistics for 14 Rex Chert and cherty shale samples collected from measured sections 1

\begin{tabular}{|c|c|c|c|c|c|c|c|}
\hline & & $\overline{\mathbf{N}}$ & Mean & Median & SD $^{T}$ & Minimum & Maximum \\
\hline $\mathrm{SiO}_{2}$ & wt $\%$ & 14 & 92.2 & 93.1 & 6.28 & 78.7 & 99.9 \\
\hline $\mathrm{Al}_{2} \mathrm{O}_{3}$ & & 14 & 2.23 & 1.80 & 2.18 & 0.26 & 8.41 \\
\hline $\mathrm{Fe}_{2} \mathrm{O}_{3}$ & & 14 & 1.17 & 0.77 & 1.24 & 0.17 & 4.68 \\
\hline $\mathrm{TiO}_{2}$ & & 14 & 0.13 & 0.08 & 0.15 & 0.01 & 0.55 \\
\hline $\mathrm{CaO}$ & & 14 & 1.32 & 1.05 & 0.96 & 0.21 & 3.69 \\
\hline $\mathrm{K}_{2} \mathrm{O}$ & & 14 & 0.43 & 0.26 & 0.51 & 0.02 & 1.95 \\
\hline $\mathrm{MgO}$ & & 14 & 0.42 & 0.25 & 0.39 & 0.01 & 1.09 \\
\hline $\mathrm{Na}_{2} \mathrm{O}$ & & 14 & 0.24 & 0.07 & 0.25 & 0.01 & 0.63 \\
\hline $\mathrm{P}_{2} \mathrm{O}_{5}$ & & 14 & 0.62 & 0.41 & 0.74 & 0.09 & 3.05 \\
\hline$\sum \mathrm{CO}_{2}$ & & 14 & 0.56 & 0.06 & 0.77 & 0.01 & 2.15 \\
\hline Total & & 14 & 99.3 & 99.6 & 1.5 & 95.4 & 101 \\
\hline $\mathrm{C}_{\mathrm{t}}$ & & 14 & 0.63 & 0.39 & 0.58 & 0.01 & 1.81 \\
\hline $\mathrm{C}_{\mathrm{c}}$ & & 14 & 0.15 & 0.02 & 0.21 & 0.0015 & 0.59 \\
\hline $\mathrm{C}_{\text {org }}$ & & 14 & 0.48 & 0.31 & 0.50 & 0.007 & 1.8 \\
\hline $\mathrm{S}_{\mathrm{t}}$ & & 14 & 0.05 & 0.03 & 0.05 & 0.025 & 0.2 \\
\hline As & ppm & 14 & 4.3 & 3.1 & 4.0 & 0.7 & 13.2 \\
\hline $\mathrm{Ba}$ & & 14 & 142 & 68 & 179 & 18 & 593 \\
\hline $\mathrm{Ba}$ & & 14 & 140 & 70 & 177 & 16 & 611 \\
\hline $\mathrm{Ce}$ & & 14 & 17 & 11 & 22 & 2.5 & 87 \\
\hline $\mathrm{Cr}$ & & 14 & 239 & 138 & 310 & 19 & 1100 \\
\hline $\mathrm{Cu}$ & & 14 & 38 & 31 & 35 & 11 & 152 \\
\hline $\mathrm{Hg}$ & & 14 & 0.05 & 0.04 & 0.04 & 0.01 & 0.15 \\
\hline $\mathrm{La}$ & & 14 & 25 & 15 & 44 & 3 & 176 \\
\hline $\mathrm{Li}$ & & 14 & 14 & 11 & 11 & 4 & 43 \\
\hline $\mathrm{Mn}$ & & 14 & 68 & 46 & 80 & 13 & 333 \\
\hline $\mathrm{Nd}$ & & 14 & 31 & 22 & 49 & 4.5 & 197 \\
\hline $\mathrm{Ni}$ & & 14 & 29 & 21 & 24 & 4 & 97 \\
\hline $\mathrm{Sb}$ & & 14 & 0.6 & 0.3 & 0.5 & 0.3 & 2.2 \\
\hline $\mathrm{Se}$ & & 14 & 1.3 & 1.1 & 0.8 & 0.1 & 2.8 \\
\hline $\mathrm{Sr}$ & & 14 & 54 & 39 & 51 & 14 & 215 \\
\hline $\mathrm{V}$ & & 14 & 39 & 26 & 47 & 4 & 188 \\
\hline $\mathrm{Y}$ & & 14 & 48 & 31 & 80 & 6 & 321 \\
\hline $\mathrm{Zn}$ & & 14 & 88 & 90 & 38 & 30 & 154 \\
\hline $\mathrm{Zr}$ & & 14 & 51 & 30 & 49 & 5 & 164 \\
\hline
\end{tabular}

${ }^{1}$ Standard Deviation 
Table 6. Statistics for 4 Rex Chert and cherty shale samples collected from measured sections 5

\begin{tabular}{|c|c|c|c|c|c|c|}
\hline & $\overline{\mathbf{N}}$ & Mean & Median & $\mathbf{S D}^{\mathbf{T}}$ & Minimum & Maximum \\
\hline $\mathrm{SiO}_{2} \quad$ wt $\%$ & 4 & 96.9 & 97.5 & 2.05 & 93.9 & 98.6 \\
\hline $\mathrm{Al}_{2} \mathrm{O}_{3}$ & 4 & 1.05 & 1.07 & 0.39 & 0.55 & 1.49 \\
\hline $\mathrm{Fe}_{2} \mathrm{O}_{3}$ & 4 & 0.98 & 0.48 & 1.12 & 0.31 & 2.65 \\
\hline $\mathrm{TiO}_{2}$ & 4 & 0.05 & 0.04 & 0.03 & 0.017 & 0.08 \\
\hline $\mathrm{CaO}$ & 4 & 0.30 & 0.27 & 0.09 & 0.22 & 0.43 \\
\hline $\mathrm{K}_{2} \mathrm{O}$ & 4 & 0.15 & 0.17 & 0.05 & 0.07 & 0.18 \\
\hline $\mathrm{MgO}$ & 4 & 0.09 & 0.09 & 0.03 & 0.05 & 0.12 \\
\hline $\mathrm{Na}_{2} \mathrm{O}$ & 4 & 0.05 & 0.05 & 0.01 & 0.04 & 0.07 \\
\hline $\mathrm{P}_{2} \mathrm{O}_{5}$ & 4 & 0.19 & 0.16 & 0.08 & 0.14 & 0.3 \\
\hline$\sum \mathrm{CO}_{2}$ & 4 & 0.02 & 0.02 & 0.01 & 0.01 & 0.03 \\
\hline Total & 4 & 100 & 100 & 0.37 & 99.2 & 100 \\
\hline $\mathrm{C}_{\mathrm{t}}$ & 4 & 0.13 & 0.12 & 0.04 & 0.09 & 0.19 \\
\hline $\mathrm{C}_{\mathrm{c}}$ & 4 & 0.01 & 0.01 & 0.00 & 0.0015 & 0.01 \\
\hline $\mathrm{C}_{\mathrm{org}}$ & 4 & 0.12 & 0.11 & 0.05 & 0.08 & 0.187 \\
\hline $\mathrm{S}_{\mathrm{t}}$ & 4 & 0.03 & 0.03 & 0.01 & 0.025 & 0.05 \\
\hline ppm & 4 & 5.3 & 2.6 & 6.2 & 1.6 & 14.5 \\
\hline $\mathrm{Ba}$ & 4 & 227 & 200 & 154 & 85 & 423 \\
\hline $\mathrm{Ba}$ & 4 & 219 & 188 & 152 & 84 & 415 \\
\hline $\mathrm{Ce}$ & 4 & 5 & 6 & 2 & 2.5 & 8 \\
\hline $\mathrm{Cr}$ & 4 & 70 & 73 & 13 & 51 & 81 \\
\hline $\mathrm{Cu}$ & 4 & 32 & 28 & 20 & 13 & 60 \\
\hline $\mathrm{Hg}$ & 4 & 0.05 & 0.03 & 0.04 & 0.02 & 0.1 \\
\hline $\mathrm{La}$ & 4 & 6 & 6 & 3 & 4 & 10 \\
\hline $\mathrm{Li}$ & 4 & 10 & 10 & 3 & 6 & 13 \\
\hline $\mathrm{Mn}$ & 4 & 449 & 33 & 841 & 22 & 1710 \\
\hline $\mathrm{Nd}$ & 4 & 10 & 10 & 4 & 4.5 & 15 \\
\hline $\mathrm{Ni}$ & 4 & 33 & 12 & 44 & 8 & 98 \\
\hline $\mathrm{Sb}$ & 4 & 0.8 & 0.8 & 0.2 & 0.7 & 1.0 \\
\hline $\mathrm{Se}$ & 4 & 0.7 & 0.6 & 0.2 & 0.5 & 0.9 \\
\hline $\mathrm{Sr}$ & 4 & 30 & 24 & 14 & 22 & 51 \\
\hline V & 4 & 20 & 17 & 11 & 11 & 35 \\
\hline Y & 4 & 14 & 13 & 4 & 10 & 20 \\
\hline $\mathrm{Zn}$ & 4 & 92 & 48 & 102 & 29 & 244 \\
\hline $\mathrm{Zr}$ & 4 & 25 & 25 & 16 & 5 & 45 \\
\hline
\end{tabular}

${ }^{1}$ Standard Deviation 
Table 7. Statistics for 21 Rex Chert and cherty shale samples collected from measured sections 7

\begin{tabular}{|c|c|c|c|c|c|c|}
\hline & $\mathbf{N}$ & Mean & Median & $\mathbf{S D}^{1}$ & Minimum & $\overline{\text { Maximum }}$ \\
\hline $\mathrm{SiO}_{2} \quad$ wt $\%$ & 21 & 95.9 & 96.7 & 7.38 & 74.9 & 102 \\
\hline $\mathrm{Al}_{2} \mathrm{O}_{3}$ & 21 & 1.69 & 0.43 & 3.15 & 0.23 & 11.1 \\
\hline $\mathrm{Fe}_{2} \mathrm{O}_{3}$ & 21 & 0.34 & 0.14 & 0.51 & 0.04 & 2.09 \\
\hline $\mathrm{TiO}_{2}$ & 21 & 0.11 & 0.02 & 0.23 & 0.01 & 0.82 \\
\hline $\mathrm{CaO}$ & 21 & 0.70 & 0.41 & 0.74 & 0.17 & 2.92 \\
\hline $\mathrm{K}_{2} \mathrm{O}$ & 21 & 0.34 & 0.05 & 0.76 & 0.005 & 2.58 \\
\hline $\mathrm{MgO}$ & 21 & 0.16 & 0.03 & 0.24 & 0.01 & 0.7 \\
\hline $\mathrm{Na}_{2} \mathrm{O}$ & 21 & 0.18 & 0.07 & 0.36 & 0.03 & 1.32 \\
\hline $\mathrm{P}_{2} \mathrm{O}_{5}$ & 21 & 0.33 & 0.23 & 0.39 & 0.05 & 1.72 \\
\hline$\sum \mathrm{CO}_{2}$ & 21 & 0.21 & 0.02 & 0.64 & 0.005 & 2.78 \\
\hline Total & 21 & 99.9 & 101 & 3.2 & 93.1 & 103 \\
\hline $\mathrm{C}_{\mathrm{t}}$ & 21 & 0.34 & 0.12 & 0.73 & 0.01 & 3.35 \\
\hline $\mathrm{C}_{\mathrm{c}}$ & 21 & 0.06 & 0.01 & 0.17 & 0.0015 & 0.76 \\
\hline $\mathrm{C}_{\text {org }}$ & 21 & 0.28 & 0.08 & 0.72 & 0.007 & 3.347 \\
\hline $\mathrm{S}_{\mathrm{t}}$ & 21 & 0.05 & 0.03 & 0.09 & 0.025 & 0.42 \\
\hline ppm & 21 & 4.0 & 2.2 & 4.6 & 0.3 & 16.8 \\
\hline $\mathrm{Ba}$ & 21 & 70 & 37 & 97 & 17 & 365 \\
\hline $\mathrm{Ba}$ & 21 & 70 & 39 & 93 & 22 & 351 \\
\hline $\mathrm{Ce}$ & 21 & 8 & 3 & 13 & 2.5 & 47 \\
\hline $\mathrm{Cr}$ & 21 & 93 & 16 & 158 & 5 & 601 \\
\hline $\mathrm{Cu}$ & 21 & 54 & 33 & 73 & 5 & 296 \\
\hline $\mathrm{Hg}$ & 21 & 0.03 & 0.01 & 0.04 & 0.01 & 0.16 \\
\hline $\mathrm{La}$ & 21 & 12 & 6 & 12 & 3 & 48 \\
\hline $\mathrm{Li}$ & 21 & 8 & 6 & 6 & 1 & 21 \\
\hline Mn & 21 & 107 & 58 & 85 & 21 & 299 \\
\hline $\mathrm{Nd}$ & 21 & 9 & 5 & 9 & 4.5 & 37 \\
\hline $\mathrm{Ni}$ & 21 & 20 & 15 & 19 & 4 & 86 \\
\hline $\mathrm{Sb}$ & 21 & 1.1 & 0.3 & 2.4 & 0.3 & 11.4 \\
\hline $\mathrm{Se}$ & 21 & 12.1 & 0.2 & 36.2 & 0.1 & 138 \\
\hline $\mathrm{Sr}$ & 21 & 30 & 19 & 26 & 8 & 95 \\
\hline V & 21 & 25 & 5 & 39 & 1 & 130 \\
\hline Y & 21 & 14 & 8 & 17 & 3 & 76 \\
\hline $\mathrm{Zn}$ & 21 & 128 & 91 & 136 & 24 & 569 \\
\hline $\mathrm{Zr}$ & 21 & 51 & 13 & 115 & 5 & 397 \\
\hline
\end{tabular}

${ }^{1}$ Standard Deviation 
$12 \mathrm{ppm}$ is heavily dependent on two sample values of 101 and $138 \mathrm{ppm}$. Without those two samples, the mean Se concentration in section 7 would be $0.8 \mathrm{ppm} \mathrm{Se}$, close to its crustal mean content (Table 3).

Other elements of environmental interest include $\mathrm{As}, \mathrm{Cr}, \mathrm{V}, \mathrm{Zn}, \mathrm{Hg}$, and $\mathrm{Cd}$. Arsenic concentrations vary by a factor of 56 , from 0.3 to $16.8 \mathrm{ppm}$, mean $4.2 \mathrm{ppm}$, which is slightly less than the concentration in average shale of $6.6 \mathrm{ppm}$ (Govett, 1983). Chromium concentrations vary by a factor of 220 , from 5 to $1100 \mathrm{ppm}$, with a mean of $143 \mathrm{ppm}$, which is higher than the $100 \mathrm{ppm}$ concentration in average shale. Vanadium concentrations vary by a factor of 188 , from 1 to $188 \mathrm{ppm}$, mean $30 \mathrm{ppm}$, which is much lower than the $130 \mathrm{ppm}$ concentration in average shale. Zinc concentrations vary by a factor of 24 , from 24 to $569 \mathrm{ppm}$, mean $110 \mathrm{ppm}$, which is somewhat greater than the concentration in average shale of $80 \mathrm{ppm}$. Mercury concentrations vary by a factor of $>8$, from 0.01 to $0.16 \mathrm{ppm}$, mean $0.04 \mathrm{ppm}$, which is an order of magnitude less than the concentration in average shale of $0.4 \mathrm{ppm}$. Cadmium concentrations are uniformly below the limit of quantification $(<2 \mathrm{ppm})$ except for rocks from the lowermost part of section 7 ; we are unable to ascertain whether the average may be higher than that of average shale, $0.3 \mathrm{ppm}$.

\section{Stratigraphic Changes in Chemical Composition}

Stratigraphic changes (equivalent to temporal changes in the depositional basin) in chemical composition of rocks are notable either as uniform changes through the sections or as distinct differences in the mean composition of rocks that compose the upper and lower halves of the sections. In this regard, most elements increase up section in section 1, whereas they decrease up section in sections 5 and 7 . These increases up section in section 1 still occur for about half of the elements if the cherty shale member is not included in the analysis; the other half of the elements do not vary uniformly up section. Silica has the opposite trend of the other elements. For section 1 (including the cherty shale member), the following elements increase up section: $\mathrm{Al}, \mathrm{Fe}, \mathrm{Ti}, \mathrm{K}, \mathrm{Na}, \mathrm{As}, \mathrm{Ba}, \mathrm{Ce}, \mathrm{Cr}, \mathrm{Hg}, \mathrm{La}$, $\mathrm{Li}, \mathrm{Ni}, \mathrm{Sc}, \mathrm{Sr}, \mathrm{V}$, and $\mathrm{Zr}$; in contrast, silica decreases up section and $\mathrm{Ca}, \mathrm{Mg}, \mathrm{C}$, and $\mathrm{Mn}$ decrease to near mid-section then increase farther up section. For section 5, the following elements decrease up section: Al, Fe, Ti, K, Na, C, Ce, Ni, Sr, V, Y, and Zn; whereas Si increases up section. For section 7, the following elements decrease up section: $\mathrm{Al}, \mathrm{Fe}$, Ti, K, Na, organic C, Ba, Ce, Cr, La, Li, Ni, Se, Sr, Tl, V, Y, Zn, and Zr; whereas Si and $\mathrm{Mn}$ increase up section.

\section{Phase Associations of Elements}

The phase associations of elements were determined by comparing results from element correlations determined from correlation coefficient matrices (Tables 8-10), rotated factor loadings from Q-mode factor analyses (Figures 3-5), and mineralogy as determined by XRD (Table 2). Three data sets were analyzed including data from sections 1,5 , and 7 combined, section 1 data, and section 7 data (excluding Meade Peak and Rex Chert carbonate beds).

We consider four to five Q-mode factors that are interpreted to represent the following rock and mineral components: Factor 1, Chert-silica component consisting solely of Si, except for the combined data set where Ba shows a minor but statistically significant factor loading. Factor 2, phosphorite-phosphate component comprised of $\mathrm{P}, \mathrm{Ca}, \mathrm{As}, \mathrm{Y}, \mathrm{V}, \mathrm{Cr}$, $\mathrm{Sr}$, and $\mathrm{La}( \pm \mathrm{Fe}, \mathrm{Zn}, \mathrm{Cu}, \mathrm{Ni}, \mathrm{Li}, \mathrm{Se}, \mathrm{Nd}$, and $\mathrm{Hg}$ depending on the data set). Factor 3, shale component comprised of $\mathrm{Al}, \mathrm{Na}, \mathrm{Zr}, \mathrm{K}, \mathrm{Ba}, \mathrm{Li}$, and organic $\mathrm{C}( \pm \mathrm{Ti}, \mathrm{Mg}, \mathrm{Se}, \mathrm{Ni}$, $\mathrm{Fe}, \mathrm{Sr}, \mathrm{V}, \mathrm{Mn}$, and $\mathrm{Zn}$ depending on the data set). Factor 4, carbonate component (dolomite, calcite, silicified carbonates) comprised of carbonate $\mathrm{C}, \mathrm{Mg}, \mathrm{Ca}$, and $\mathrm{Si}( \pm \mathrm{Mn})$. Factor 5 we tentatively interpret as representing organic matter (and/or sulfide-sulfate phases) hosted elements comprised of $\mathrm{Cu}$ ( \pm organic $\mathrm{C}, \mathrm{Zn}, \mathrm{Mn} \mathrm{Si}, \mathrm{Ni}, \mathrm{Hg}$, and $\mathrm{Li}$ depending on the data set). Copper correlates only with $\mathrm{Zn}$, but $\mathrm{Zn}$ also correlates with $\mathrm{Ni}$, As and some elements associated with the shale component. Silica is dominantly in the 
Table 8. Correlation coefficient matrix for 39 samples from sections 1, 5, and 7 combined listed in Table 3; the point of zero correlation for $\mathrm{n}=39$ at the $99 \%$ confidence level is $10.4047 \mid$

\begin{tabular}{|c|c|c|c|c|c|c|c|c|c|c|c|c|c|c|c|c|c|c|c|c|}
\hline & $\mathrm{SiO}_{2}$ & $\overline{\mathrm{Al}_{2} \mathrm{O}_{3}}$ & $\mathrm{Fe}_{2} \mathrm{O}_{3}$ & $\mathrm{TiO}_{2}$ & $\mathrm{CaO}$ & $\mathrm{K}_{2} \mathrm{O}$ & $\mathrm{MgO}$ & $\mathrm{Na}_{2} \mathrm{O}$ & $\mathrm{P}_{2} \mathrm{O}_{5}$ & $\mathrm{ZCO}_{2}$ & $\mathrm{C}_{\mathrm{T}}$ & $\mathrm{C}_{\mathrm{C}}$ & $\mathrm{C}_{\text {org }}$ & As & $\mathrm{Ba}$ & $\mathrm{Cr}$ & $\mathrm{Cu}$ & $\mathrm{La}$ & $\mathrm{Li}$ & $\mathrm{Mn}$ \\
\hline $\mathrm{Al}_{2} \mathrm{O}_{3}$ & -0.900 & & & & & & & & & & & & & & & & & & & \\
\hline $\mathrm{Fe}_{2} \mathrm{O}_{3}$ & -0.714 & 0.553 & & & & & & & & & & & & & & & & & & \\
\hline $\mathrm{TiO}_{2}$ & -0.878 & 0.995 & 0.496 & & & & & & & & & & & & & & & & & \\
\hline $\mathrm{CaO}$ & -0.371 & 0.065 & 0.471 & 0.022 & & & & & & & & & & & & & & & & \\
\hline $\mathrm{K}_{2} \mathrm{O}$ & -0.893 & 0.995 & 0.553 & 0.992 & 0.062 & & & & & & & & & & & & & & & \\
\hline $\mathrm{MgO}$ & -0.674 & 0.556 & 0.382 & 0.550 & 0.626 & 0.544 & & & & & & & & & & & & & & \\
\hline $\mathrm{Na}_{2} \mathrm{O}$ & -0.824 & 0.939 & 0.419 & 0.953 & -0.003 & 0.916 & 0.570 & & & & & & & & & & & & & \\
\hline $\mathrm{P}_{2} \mathrm{O}_{5}$ & -0.349 & 0.092 & 0.737 & 0.021 & 0.682 & 0.099 & 0.111 & -0.080 & & & & & & & & & & & & \\
\hline$\sum \mathrm{CO}_{2}$ & -0.113 & -0.045 & -0.103 & -0.031 & 0.647 & -0.054 & 0.742 & 0.045 & -0.108 & & & & & & & & & & & \\
\hline $\mathrm{C}_{\mathrm{T}}$ & -0.782 & 0.763 & 0.415 & 0.768 & 0.223 & 0.772 & 0.739 & 0.737 & -0.042 & 0.342 & & & & & & & & & & \\
\hline $\mathrm{C}_{S}^{\mathrm{T}}$ & -0.111 & -0.048 & -0.105 & -0.034 & 0.645 & -0.058 & 0.739 & 0.042 & -0.110 & 1.000 & 0.340 & & & & & & & & & \\
\hline $\mathrm{C}_{\mathrm{org}}$ & -0.797 & 0.824 & 0.472 & 0.825 & 0.044 & 0.836 & 0.564 & 0.769 & -0.011 & 0.065 & 0.960 & 0.063 & & & & & & & & \\
\hline As & -0.610 & 0.516 & 0.697 & 0.457 & 0.382 & 0.522 & 0.240 & 0.315 & 0.670 & -0.196 & 0.415 & -0.199 & 0.501 & & & & & & & \\
\hline $\mathrm{Ba}$ & -0.544 & 0.565 & 0.247 & 0.570 & 0.094 & 0.547 & 0.553 & 0.612 & -0.062 & 0.170 & 0.517 & 0.166 & 0.499 & 0.164 & & & & & & \\
\hline $\mathrm{Cr}$ & -0.717 & 0.537 & 0.926 & 0.472 & 0.565 & 0.547 & 0.404 & 0.331 & 0.813 & -0.076 & 0.425 & $\begin{array}{r}-0.080 \\
\end{array}$ & 0.475 & 0.826 & 0.230 & & & & & \\
\hline $\mathrm{Cu}$ & 0.032 & 0.030 & -0.007 & 0.016 & 0.016 & 0.030 & -0.115 & -0.013 & 0.147 & -0.164 & -0.044 & -0.168 & 0.003 & 0.255 & -0.097 & 0.095 & & & & \\
\hline $\mathrm{La}$ & -0.501 & 0.277 & 0.849 & 0.225 & 0.618 & 0.298 & 0.221 & 0.130 & 0.909 & -0.092 & 0.130 & -0.095 & 0.166 & 0.620 & 0.081 & 0.864 & 0.061 & & & \\
\hline $\mathrm{Li}$ & -0.705 & 0.587 & 0.913 & 0.535 & 0.426 & 0.565 & 0.386 & 0.500 & 0.660 & -0.106 & 0.368 & -0.110 & 0.423 & 0.668 & 0.356 & 0.854 & 0.118 & 0.770 & & \\
\hline Mn & 0.274 & -0.182 & -0.126 & -0.163 & -0.157 & -0.214 & -0.113 & -0.051 & -0.222 & 0.030 & -0.183 & 0.030 & -0.204 & -0.253 & -0.233 & -0.225 & 0.028 & -0.211 & -0.008 & \\
\hline $\mathrm{Ni}$ & -0.626 & 0.572 & 0.497 & 0.518 & 0.361 & 0.534 & 0.448 & 0.442 & 0.377 & 0.046 & 0.448 & 0.043 & 0.463 & 0.675 & 0.295 & 0.645 & 0.242 & 0.267 & 0.585 & 0.052 \\
\hline $\mathrm{Se}$ & -0.643 & 0.806 & 0.262 & 0.838 & -0.160 & 0.827 & 0.290 & 0.815 & -0.131 & -0.108 & 0.664 & -0.110 & 0.737 & 0.304 & 0.402 & 0.197 & -0.007 & 0.111 & 0.271 & -0.176 \\
\hline $\mathrm{Sr}$ & -0.717 & 0.502 & 0.846 & 0.453 & 0.669 & 0.508 & 0.476 & 0.382 & 0.823 & 0.051 & 0.371 & 0.046 & 0.380 & 0.671 & 0.409 & 0.883 & 0.050 & 0.916 & 0.816 & -0.315 \\
\hline $\mathrm{V}$ & -0.756 & 0.612 & 0.872 & 0.557 & 0.526 & 0.623 & 0.356 & 0.433 & 0.796 & -0.126 & 0.442 & -0.130 & 0.509 & 0.874 & 0.259 & 0.940 & 0.165 & 0.855 & 0.832 & -0.284 \\
\hline Y & -0.384 & 0.127 & 0.822 & 0.068 & 0.647 & 0.144 & 0.169 & -0.018 & 0.938 & -0.070 & 0.019 & -0.073 & 0.043 & 0.550 & 0.011 & 0.823 & 0.041 & 0.977 & 0.719 & -0.193 \\
\hline $\mathrm{Zn}$ & -0.195 & 0.187 & 0.132 & 0.141 & 0.292 & 0.162 & 0.029 & 0.072 & 0.415 & -0.101 & 0.004 & -0.104 & 0.035 & 0.609 & -0.031 & 0.347 & 0.620 & 0.176 & 0.253 & -0.014 \\
\hline $\mathrm{Zr}$ & -0.823 & 0.963 & 0.420 & 0.980 & -0.039 & 0.963 & 0.500 & 0.958 & -0.050 & -0.035 & 0.746 & -0.038 & 0.803 & 0.369 & 0.557 & 0.365 & -0.007 & 0.180 & 0.463 & -0.157 \\
\hline
\end{tabular}

\begin{tabular}{llrllll}
\hline & $\mathrm{Ni}$ & \multicolumn{1}{c}{$\mathrm{Se}$} & $\mathrm{Sr}$ & $\mathrm{V}$ & $\mathrm{Y}$ & $\mathrm{Zn}$ \\
\hline $\mathrm{Se}$ & 0.101 & & & & & \\
$\mathrm{Sr}$ & 0.434 & 0.271 & & & & \\
$\mathrm{~V}$ & 0.581 & 0.411 & 0.902 & & & \\
$\mathrm{Y}$ & 0.221 & -0.062 & 0.868 & 0.778 & & \\
$\mathrm{Zn}$ & 0.665 & 0.012 & 0.251 & 0.457 & 0.143 & \\
$\mathrm{Zr}$ & 0.369 & 0.915 & 0.403 & 0.495 & 0.015 & 0.069 \\
\hline
\end{tabular}


Table 9. Correlation coefficient matrix for 14 samples from section 1 listed in Table 3; the point of zero correlation for $\mathrm{n}=14$ at the $99 \%$ confidence level is $|0.651|$

\begin{tabular}{|c|c|c|c|c|c|c|c|c|c|c|c|c|c|c|c|c|c|c|c|c|c|c|}
\hline & $\mathrm{SiO}_{2}$ & $\overline{\mathrm{Al}_{2} \mathrm{O}_{3}}$ & $\mathrm{Fe}_{2} \mathrm{O}_{3}$ & $\mathrm{TiO}_{2}$ & $\mathrm{CaO}$ & $\overline{\mathrm{K}_{2} \mathrm{O}}$ & $\overline{\mathrm{MgO}}$ & $\mathrm{Na}_{2} \mathrm{O}$ & $\mathrm{P}_{2} \mathrm{O}_{5}$ & $\Sigma \mathrm{CO}_{2}$ & $\overline{\mathrm{C}_{\mathrm{T}}}$ & $\overline{\mathrm{Cc}}$ & $\mathrm{C}_{\text {org }}$ & $\overline{\mathrm{As}}$ & $\overline{\mathrm{Ba}}$ & $\mathrm{Ce}$ & $\mathrm{Cr}$ & $\mathrm{Cu}$ & $\overline{\mathrm{Hg}}$ & $\overline{\mathrm{La}}$ & $\mathrm{Li}$ & $\mathrm{Mn}$ \\
\hline $\mathrm{Al}_{2} \mathrm{O}_{3}$ & -0.884 & & & & & & & & & & & & & & & & & & & & & \\
\hline $\mathrm{Fe}_{2} \mathrm{O}_{3}$ & -0.717 & 0.514 & & & & & & & & & & & & & & & & & & & & \\
\hline $\mathrm{TiO}_{2}$ & -0.858 & 0.993 & 0.436 & & & & & & & & & & & & & & & & & & & \\
\hline $\mathrm{CaO}^{2}$ & -0.573 & 0.136 & 0.523 & 0.105 & & & & & & & & & & & & & & & & & & \\
\hline $\mathrm{K}_{2} \mathrm{O}$ & -0.895 & 0.984 & 0.549 & 0.968 & 0.190 & & & & & & & & & & & & & & & & & \\
\hline $\mathrm{MgO}$ & -0.697 & 0.548 & 0.132 & 0.580 & 0.608 & 0.535 & & & & & & & & & & & & & & & & \\
\hline $\mathrm{Na}_{2} \mathrm{O}$ & -0.666 & 0.784 & 0.234 & 0.814 & 0.055 & 0.672 & 0.592 & & & & & & & & & & & & & & & \\
\hline $\mathrm{P}_{2} \mathrm{O}_{5}$ & -0.476 & 0.134 & 0.851 & 0.050 & 0.704 & 0.212 & 0.012 & -0.148 & & & & & & & & & & & & & & \\
\hline$\Sigma \mathrm{CO}_{2}$ & -0.181 & -0.004 & -0.322 & 0.053 & 0.502 & -0.027 & 0.806 & 0.233 & -0.257 & & & & & & & & & & & & & \\
\hline $\mathrm{C}_{\mathrm{T}}$ & -0.754 & 0.774 & 0.101 & 0.800 & 0.322 & 0.776 & 0.863 & 0.669 & -0.114 & 0.551 & & & & & & & & & & & & \\
\hline $\mathrm{Cc}$ & -0.175 & -0.008 & -0.326 & 0.048 & 0.498 & -0.032 & 0.803 & 0.229 & -0.261 & 1.000 & 0.547 & & & & & & & & & & & \\
\hline $\mathrm{C}_{\mathrm{org}}$ & -0.805 & 0.906 & 0.254 & 0.912 & 0.167 & 0.918 & 0.670 & 0.683 & -0.023 & 0.224 & 0.937 & 0.220 & & & & & & & & & & \\
\hline $\mathrm{As}^{\text {org }}$ & -0.738 & 0.644 & 0.907 & 0.566 & 0.383 & 0.707 & 0.151 & 0.220 & 0.732 & -0.377 & 0.236 & -0.379 & 0.434 & & & & & & & & & \\
\hline $\mathrm{Ba}$ & -0.432 & 0.436 & 0.002 & 0.482 & 0.247 & 0.395 & 0.654 & 0.605 & -0.103 & 0.429 & 0.547 & 0.422 & 0.461 & 0.076 & & & & & & & & \\
\hline $\mathrm{Ce}$ & -0.737 & 0.458 & 0.940 & 0.393 & 0.695 & 0.516 & 0.260 & 0.180 & 0.894 & -0.151 & 0.186 & -0.157 & 0.282 & 0.850 & 0.128 & & & & & & & \\
\hline $\mathrm{Cr}$ & -0.812 & 0.610 & 0.944 & 0.541 & 0.589 & 0.681 & 0.273 & 0.221 & 0.827 & -0.216 & 0.307 & -0.222 & 0.451 & 0.927 & 0.089 & 0.961 & & & & & & \\
\hline $\mathrm{Cu}$ & 0.007 & 0.055 & -0.051 & 0.052 & -0.215 & 0.090 & -0.107 & -0.059 & -0.117 & -0.164 & 0.122 & -0.166 & 0.212 & -0.036 & -0.070 & -0.026 & 0.058 & & & & & \\
\hline $\mathrm{Hg}$ & -0.802 & 0.700 & 0.855 & 0.638 & 0.415 & 0.763 & 0.243 & 0.295 & 0.699 & -0.309 & 0.357 & -0.313 & 0.547 & 0.912 & 0.198 & 0.817 & 0.919 & 0.163 & & & & \\
\hline $\mathrm{La}$ & -0.512 & 0.167 & 0.869 & 0.094 & 0.717 & 0.244 & 0.074 & -0.097 & 0.967 & -0.204 & -0.069 & -0.209 & 0.008 & 0.741 & -0.043 & 0.946 & 0.866 & -0.061 & 0.700 & & & \\
\hline $\mathrm{Li}$ & -0.722 & 0.560 & 0.928 & 0.511 & 0.446 & 0.550 & 0.204 & 0.429 & 0.702 & -0.252 & 0.140 & -0.258 & 0.271 & 0.795 & 0.188 & 0.870 & 0.854 & -0.001 & 0.809 & 0.766 & & \\
\hline $\mathrm{Mn}$ & -0.188 & 0.274 & 0.176 & 0.291 & -0.163 & 0.121 & 0.086 & 0.648 & -0.167 & -0.010 & 0.031 & -0.008 & 0.039 & -0.023 & 0.067 & -0.026 & -0.038 & -0.100 & 0.022 & -0.137 & 0.415 & \\
\hline $\mathrm{Nd}$ & -0.517 & 0.178 & 0.883 & 0.101 & 0.708 & 0.254 & 0.063 & -0.091 & 0.976 & -0.226 & -0.074 & -0.231 & 0.011 & 0.756 & -0.037 & 0.949 & 0.871 & -0.066 & 0.709 & 0.997 & 0.768 & -0.142 \\
\hline $\mathrm{Ni}$ & -0.797 & 0.968 & 0.411 & 0.967 & -0.008 & 0.930 & 0.471 & 0.807 & 0.009 & -0.050 & 0.739 & -0.052 & 0.883 & 0.536 & 0.382 & 0.312 & 0.486 & 0.149 & 0.641 & 0.027 & 0.502 & 0.402 \\
\hline $\mathrm{Se}$ & -0.513 & 0.329 & 0.325 & 0.310 & 0.527 & 0.302 & 0.449 & 0.462 & 0.407 & 0.209 & 0.377 & 0.201 & 0.356 & 0.249 & 0.580 & 0.451 & 0.355 & -0.087 & 0.311 & 0.369 & 0.355 & 0.025 \\
\hline $\mathrm{Sr}$ & -0.677 & 0.318 & 0.823 & 0.264 & 0.843 & 0.378 & 0.355 & 0.120 & 0.904 & 0.035 & 0.188 & 0.028 & 0.207 & 0.706 & 0.251 & 0.950 & 0.862 & -0.091 & 0.715 & 0.939 & 0.766 & -0.118 \\
\hline $\mathrm{V}$ & -0.698 & 0.421 & 0.955 & 0.343 & 0.663 & 0.493 & 0.181 & 0.082 & 0.927 & -0.229 & 0.129 & -0.234 & 0.249 & 0.883 & 0.025 & 0.982 & 0.969 & 0.030 & 0.858 & 0.952 & 0.850 & -0.064 \\
\hline Y & -0.483 & 0.132 & 0.867 & 0.054 & 0.714 & 0.209 & 0.039 & -0.131 & 0.984 & -0.225 & -0.108 & -0.229 & -0.029 & 0.731 & -0.073 & 0.930 & 0.848 & -0.065 & 0.686 & 0.994 & 0.744 & -0.150 \\
\hline $\mathrm{Zn}$ & -0.393 & 0.546 & 0.148 & 0.526 & -0.176 & 0.510 & 0.214 & 0.484 & -0.136 & -0.070 & 0.455 & -0.067 & 0.559 & 0.207 & 0.108 & -0.020 & 0.143 & 0.502 & 0.337 & -0.213 & 0.135 & 0.298 \\
\hline $\mathrm{Zr}$ & -0.843 & 0.959 & 0.384 & 0.976 & 0.145 & 0.908 & 0.627 & 0.890 & 0.023 & 0.140 & 0.801 & 0.134 & 0.878 & 0.469 & 0.569 & 0.363 & 0.470 & -0.004 & 0.547 & 0.061 & 0.491 & 0.355 \\
\hline
\end{tabular}

\begin{tabular}{lrrlrlrl}
\hline & $\mathrm{Nd}$ & $\mathrm{Ni}$ & $\mathrm{Se}$ & $\mathrm{Sr}$ & $\mathrm{V}$ & $\mathrm{Y}$ & $\mathrm{Zn}$ \\
\hline $\mathrm{Ni}$ & 0.034 & & & & & & \\
$\mathrm{Se}$ & 0.391 & 0.262 & & & & & \\
$\mathrm{Sr}$ & 0.940 & 0.165 & 0.592 & & & & \\
$\mathrm{~V}$ & 0.958 & 0.292 & 0.378 & 0.920 & & & \\
$\mathrm{Y}$ & 0.998 & -0.006 & 0.382 & 0.929 & 0.947 & & \\
$\mathrm{Zn}$ & -0.175 & 0.635 & 0.106 & -0.137 & 0.047 & -0.177 & \\
$\mathrm{Zr}$ & 0.071 & 0.933 & 0.438 & 0.274 & 0.287 & 0.025 & 0.501 \\
\hline
\end{tabular}


Table 10. Correlation coefficient matrix for 21 samples from section 7 listed in Table 3; the point of zero correlation for $\mathrm{n}=21$ at the $99 \%$ confidence level is $10.542 \mid$

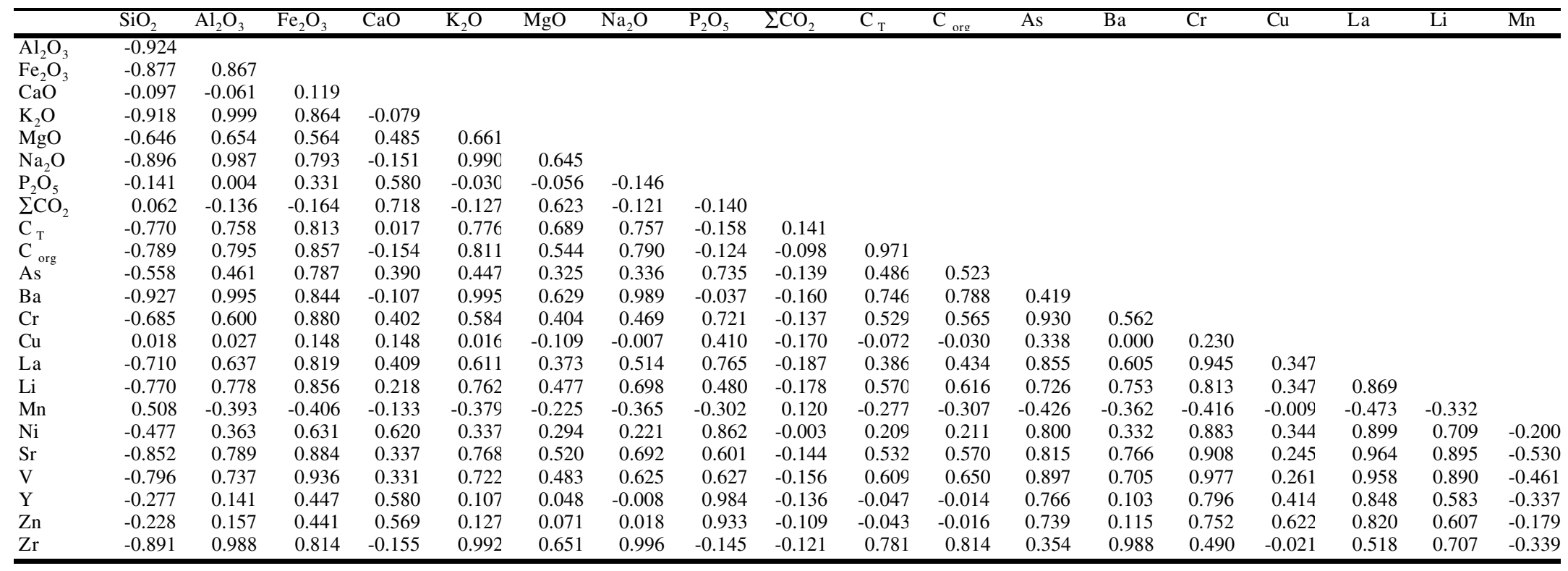

\begin{tabular}{llllrl}
\hline & $\mathrm{Ni}$ & $\mathrm{Sr}$ & $\mathrm{V}$ & $\mathrm{Y}$ & $\mathrm{Zn}$ \\
\hline $\mathrm{Sr}$ & 0.810 & & & & \\
$\mathrm{~V}$ & 0.836 & 0.963 & & & \\
$\mathrm{Y}$ & 0.916 & 0.705 & 0.722 & & \\
$\mathrm{Zn}$ & 0.910 & 0.671 & 0.696 & 0.951 & \\
$\mathrm{Zr}$ & 0.225 & 0.689 & 0.639 & -0.008 & 0.018 \\
\hline
\end{tabular}




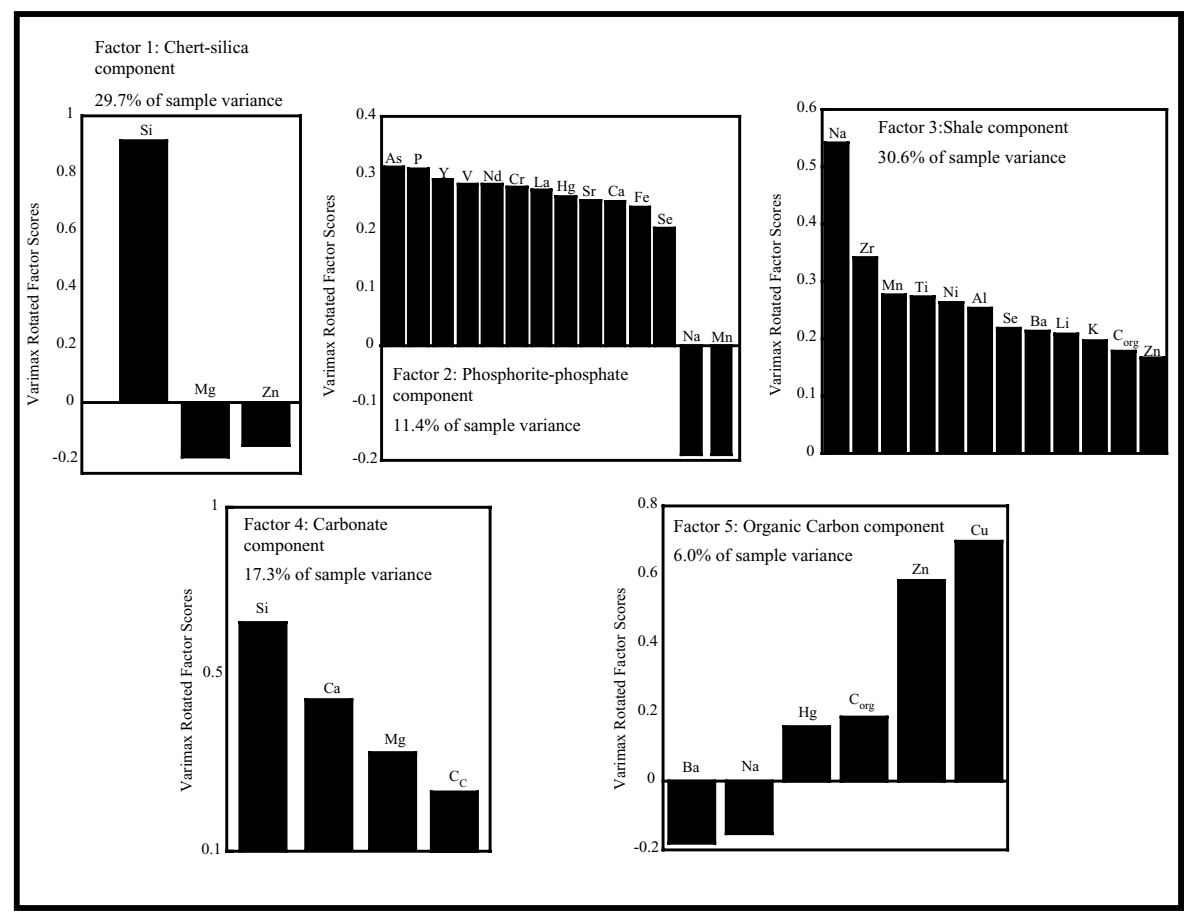

Figure 3. Q-mode factors for Rex Chert and cherty shale samples from sections 1,5 , and 7

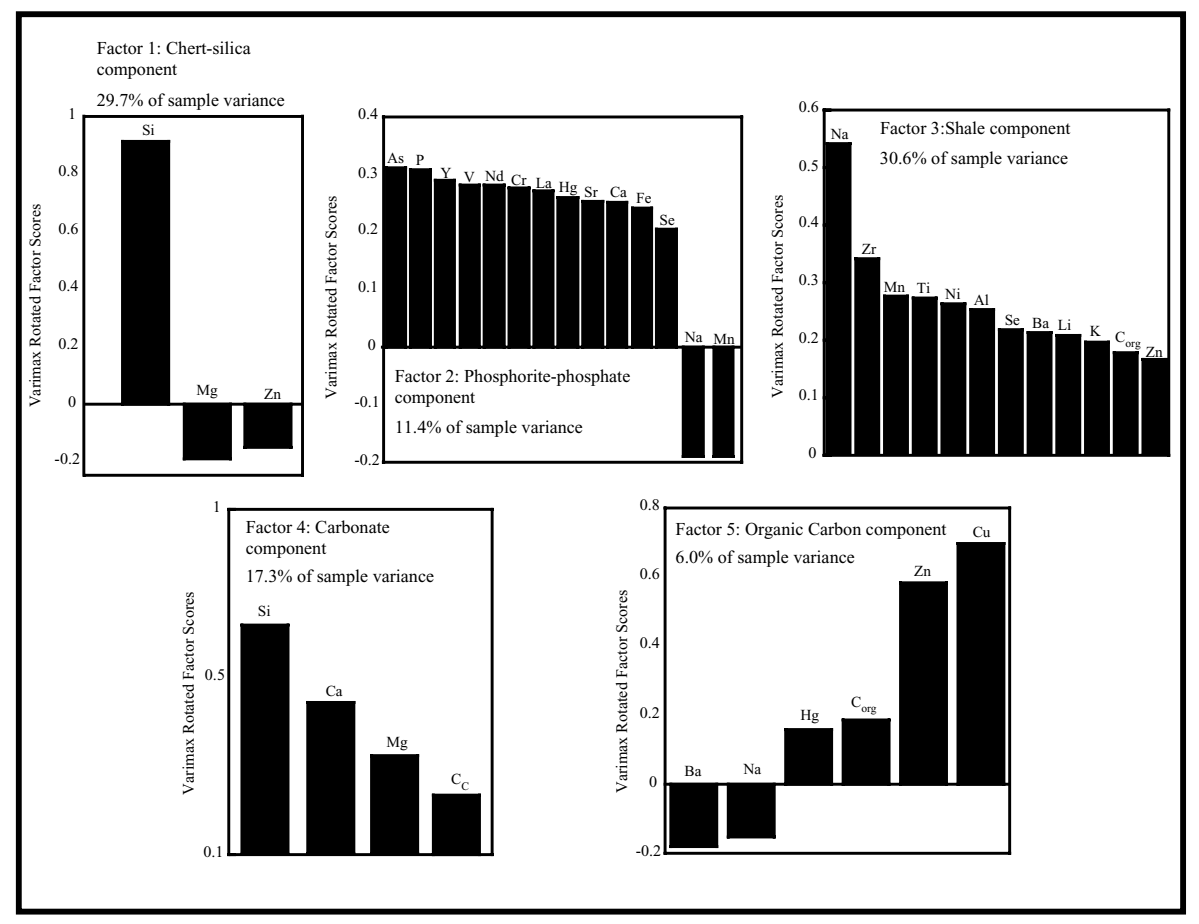

Figure 4. Q-mode factors for Rex Chert and cherty shale samples from section 1 


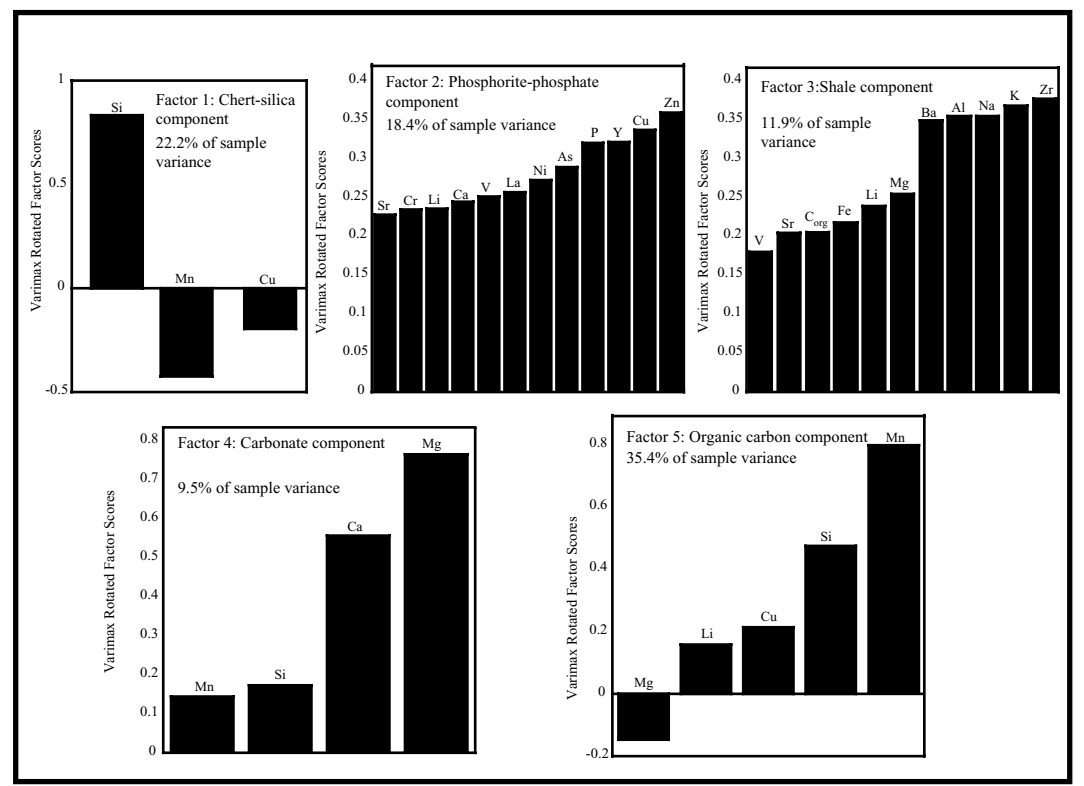

Figure 5. Q-mode factors for Rex Chert and cherty shale samples from section 7

chert fraction (factor 1) and does not show up in the aluminosilicate fraction (factor 3 shale fraction) even though it is clearly part of the feldspars and clay minerals that comprise that fraction. Likewise, organic $\mathrm{C}$ is dominantly in Factor 3 and does not show up in factor 5 (except for section 1) even though it is likely that the elements in factor 5 are hosted by organic matter. These characteristics are an artifact of analyzing a data set that is overwhelmingly dominated by one variable, silica, and the distribution of some elements in more than one phase. Selenium shows a dominant association with the shale component, but correlations and Q-mode factors also indicate that organic matter (within the shale component) and carbonate fluorapatite may host a portion of the Se. Consideration of larger numbers of factors in Q-mode analysis indicates that native Se (a factor containing Se $\pm \mathrm{Ba}$ ) may also comprise a minor component of the Se compliment.

\section{DISCUSSION AND CONCLUSIONS}

The chert beds generally have Se concentrations of $<1 \mathrm{ppm}$. The cherty shale member rocks have a somewhat higher Se contents, mean $1.8 \mathrm{ppm}$. Siliceous siltstone and argillaceous chert that comprise the zone of transition between the Meade Peak and Rex Chert have Se concentrations up to $138 \mathrm{ppm}$.

The low Se contents determined for the chert-bed samples here are not characteristic of Se concentrations found for composite channel samples of chert at the Rasmussen Ridge and Enoch Valley Mines (Herring et al., 2002). Those weighted (for stratigraphic thickness) mean Se concentrations are 63 ppm for the Rasmussen Ridge samples and 18 ppm for the Enoch Valley samples. These differences may result from several factors, such as weathering of the outcrop samples we analyzed and the inclusion of shale and siliceous shale beds that are interbedded with cherts in the composite samples. This latter influence is supported by the lower silica and higher $\mathrm{Al}_{2} \mathrm{O}_{3}, \mathrm{~K}_{2} \mathrm{O}$, etc. contents of the composite samples. In the outcrop sections studied here, shale interbedded with the chert consists only of thin partings, except in the cherty shale member, and those partings would 
not likely contribute significantly to the mean Se concentration for each outcrop section. It is not known why there are more (or thicker) shale interbeds, or more argillaceous cherts in the composite sections than those studied in outcrops. Chert is very resistant to weathering and little Se should be leached by weathering of the outcrops.

Other elements of environmental interest include $\mathrm{As}, \mathrm{Cr}, \mathrm{V}, \mathrm{Zn}, \mathrm{Hg}$, and $\mathrm{Cd}$. Of these elements, only mean $\mathrm{Cr}$ and $\mathrm{Zn}$ values are higher than their respective values in average shale, $30 \%$ and $27 \%$ higher, respectively. Cadmium could not be evaluated because most concentrations are below the limit of quantification of $2 \mathrm{ppm}$.

\section{ACKNOWLEDGMENTS}

We thank Dick Grauch for reviewing this report.

\section{REFERENCES CITED}

Arbogast, B.F., (ed.), 1996, Analytical methods manual for the Mineral Resource Surveys Program, U.S. Geological Survey: U.S. Geological Survey Open-File Report 96-525, $248 \mathrm{p}$.

Baedecker, P.A., (ed.), 1987, Methods for geochemical analysis: U.S. Geological Survey Bulletin 1770, variously paginated.

Brittenham, M.D., 1976, Permian Phosphoria carbonate banks, Idaho-Wyoming thrust belt, in Hill, J.G., ed., Symposium on geology of the Cordilleran hingeline: Rock Mountain Association of Geologists-1976 symposium, Denver, p. 173-191.

Cressman, E.R., and Swanson, R.W., 1964, Stratigraphy and petrology of the Permian rocks of southwestern Montana: U.S. Geological Survey Professional Paper 313-C, p. 275-569.

Govett, G.J.S., 1983, Handbook of Exploration Geochemistry, v. 3, Rock Geochemistry in Mineral Exploration. Elsevier, Amsterdam, 461 pp.

Herring, J.H., et. al. 2002, in preparation

Gulbrandsen, R.A., 1966, Chemical composition of phosphorites of the Phosphoria Formation: Geochimica et Cosmochimica Acta, v. 30, p. 769-778.

Gulbrandsen, R.A., 1975, Analytical data on the Phosphoria Formation, western United States: U.S. Geological Survey Open-File Report 75-554, 45 p.

Gulbrandsen, R.A., 1979, Preliminary analytical data on the Meade Peak member of the Phosphoria Formation at Hot Springs underground mine, Trail Canyon trench, and Conda underground mine, southeastern Idaho: U.S. Geological Survey Open-File Report 79-369, 35 p.

Gulbrandsen, R.A., and Krier, D.J., 1980, Large and rich phosphorus resources in the Phosphoria Formation in the Soda Springs area southeastern Idaho: U.S. Geological Survey Bulletin 1496, 25 p.

Jackson, L.L., Brown, F.W., and Neil, S.T., 1987, Major and minor elements requiring individual determinations, classical whole rock analysis, and rapid rock analysis, $\mathrm{p}$. G1-G23, in Baedecker, P.A., (ed.), Methods for geochemical analysis: U.S. Geological Survey Bulletin 1770.

Klovan J.E. and Imbrie J., 1971, An algorithm and FORTRAN-IV program for large-scale Q-mode factor analysis and calculation of factor scores. Mathematical Geology v. 3, p. 61-77. 
Krauskopf, K.B., 1979, Introduction to Geochemistry. McGraw-Hill, New York, 617 pp.

Lee, W.H., 2001, A history of phosphate mining in Southeastern Idaho. U.S. Geological Survey Open-File Report 00-425, Version 1.0, CD-ROM, 253 pp.

McKelvey, V.E., Williams, J.S., Sheldon, R.P., Cressman, E.R., Cheney, T.M., and Swanson, R.W., 1959, The Phosphoria, Park City, and Shedhorn Formations in the Western Phosphate Field: U.S. Geological Survey Professional Paper 313-A, 47 p. Montgomery, K.M, and Cheney, T.M., 1967, Geology of the Stewart Flat quadrangle, Caribou County, Idaho: U.S. Geological Bulletin 1217, 63 p.

Oberlindacher, H.P., 1990, Geologic map and phosphate resources of the northeastern part of the Lower Valley quadrangle, Caribou County, Idaho: U.S. Geological Survey Miscellaneous Field Studies Map MF-2133, scale 1:12,000.

Service, A.L., 1966, An evaluation of the western phosphate industry and its resources, Part 3. Idaho: U.S. Bureau of Mines Report of Investigations 6801, 201 p. 